In Situ

Revue des patrimoines
In Situ

Revue des patrimoines

15 | 2011

Le patrimoine des guides : lectures de l'espace urbain européen

\title{
Les Guides Joanne : invention d'une collection
}

\section{Hélène Morlier}

\section{(2) OpenEdition}

\section{Journals}

Édition électronique

URL : http://journals.openedition.org/insitu/524

DOI : 10.4000/insitu.524

ISSN : 1630-7305

Éditeur

Ministère de la culture

Référence électronique

Hélène Morlier, «Les Guides Joanne : invention d'une collection », In Situ [En ligne], 15 | 2011, mis en ligne le 29 juin 2011, consulté le 30 avril 2019. URL : http://journals.openedition.org/insitu/524 ; DOI : 10.4000/insitu.524

Ce document a été généré automatiquement le 30 avril 2019

\section{(c)}

In Situ Revues des patrimoines est mis à disposition selon les termes de la licence Creative Commons Attribution - Pas d'Utilisation Commerciale - Pas de Modification 4.0 International. 


\title{
Les Guides Joanne : invention d'une collection
}

\author{
Hélène Morlier
}

1 Éditeur de livres scolaires et de littérature, Louis Hachette s'attaque à un nouveau marché en créant, entre 1851 et 1853 , la « Bibliothèque des Chemins de fer » dont certaines séries qui, sous le nom de "Guides Joanne ", "Bibliothèque rose ", "Bibliothèque des merveilles ", étaient promises à un brillant avenir. Pour les guides de voyage, cet éditeur procède selon son habitude : il anéantit la concurrence, domine le marché et crée un poste de directeur de collection occupé par Adolphe Joanne auquel succède son fils Paul, puis un collaborateur formé depuis de longues années : Marcel Monmarché. Comme pour la direction de la Librairie Hachette, l'effet dynastique resserrait les liens de collaboration et de fidélité à l'entreprise. La collection des Guides de voyages Joanne, bien servie par des directeurs zélés, voyageurs érudits et montagnards géographes, se révèle rentable et survivra aux aléas de l'Histoire en se transformant en Guides Bleus en $1919^{1}$.

\section{Comment créer une collection de guides de voyage?}

2 En 1851, Louis Hachette se rend à Londres pour visiter, entre autres, l'Exposition universelle ${ }^{2}$. À son retour, s'inspirant de l'exemple du libraire et diffuseur W.H. Smith, il entreprend de créer une nouvelle collection de livres destinée à un large public utilisateur du nouveau moyen de transport alors en plein développement en France : les chemins de fer. C'est tout naturellement semble-t-il que le titre «Bibliothèque des Chemins de fer » s'impose, puisque le vocable « Bibliothèque du voyageur » avait déjà été pris par Napoléon Chaix, éditeur des indicateurs des chemins de fer, habituellement appelés « Indicateurs Chaix ».

Dès le départ, l'éditeur Hachette voit grand: 120 titres devaient être répartis en sept séries identifiables par la couleur de leur couverture brochée ${ }^{4}$ : la série vermillon est celle des guides, facilement repérables grâce à cette couleur vive. La particularité de cette collection est d'être diffusée dans les gares, dans des kiosques spécialement construits à cet effet dès son lancement. 
4 Très rapidement des contrats appelés «traités » sont passés avec les compagnies de chemin de fer afin d'installer les kiosques dans les stations desservies. Les contacts de Louis Hachette dans le monde des affaires et de la finance ont vraisemblablement facilité les opérations ${ }^{5}$. Le premier contrat est signé avec la compagnie du Nord en $1852^{6}$. Les autres compagnies suivent, permettant ainsi à la Librairie Hachette d'installer ses points de vente sur l'ensemble des réseaux français. Encore fallait-il approvisionner ces lieux de diffusion, trouver des auteurs et des sujets.

5 Des œuvres littéraires sont reprises, d'autres abrégées et réimprimées dans cette nouvelle collection qui voulait toucher un très large public et rester relativement bon marché avec un format pratique. Les guides de voyages présentent pour leur rédaction une difficulté spécifique : l'auteur est censé effectuer le voyage, prendre des notes précises, accumuler de la documentation et rédiger avec clarté des informations utilisables par tous. Ce sont donc des ouvrages assez longs et coûteux à mettre en œuvre. Des contrats sont établis avec quelques hommes de lettres susceptibles de rédiger rapidement les premiers titres, mais surtout les fonds de deux libraires spécialisés sont rachetés. Ernest Bourdin vend quatre titres le 25 mars 1853 à la Librairie Hachette ${ }^{7}$, dont deux sont intégrés directement ${ }^{8}$, les deux autres étant refondus en un nouveau volume le Guide du voyageur à Londres ${ }^{9}$. Le 18 juin 1855, Louis Hachette acquiert le fonds de Guides de voyages de Louis Maison qui était alors le plus important éditeur dans cette spécialité, en particulier avec ses Guides Richard (fig. $\left.\mathbf{n}^{\circ} \mathbf{1}, \mathbf{n}^{\circ} \mathbf{2}\right)^{10}$.

Figure 1

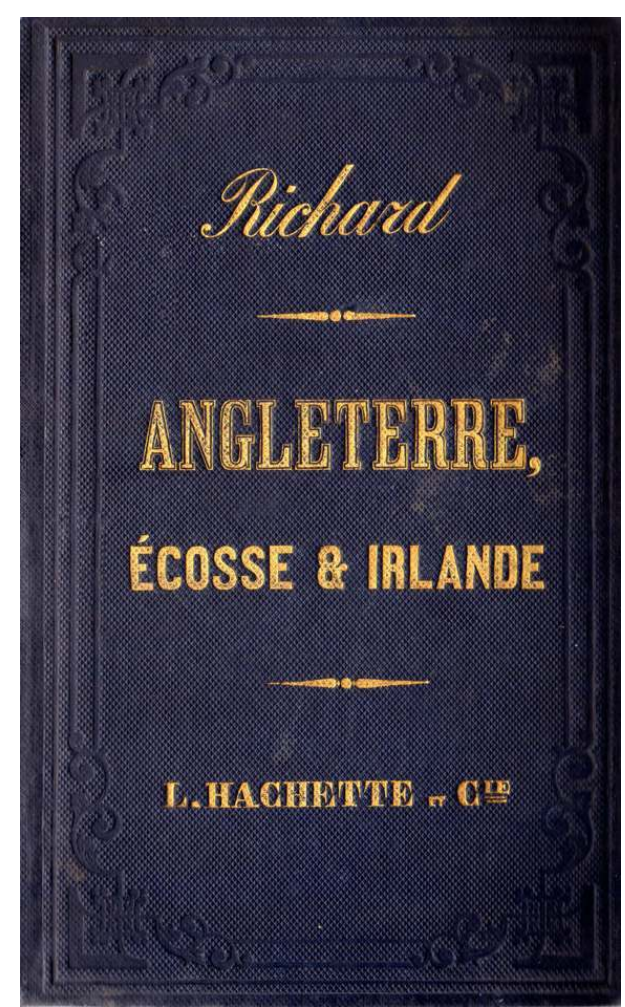

Guide Richard. Itinéraire descriptif et historique de la Grande-Bretagne. Angleterre, Écosse, Irlande. Paris : L. Maison, 1855 : couverture et page de titre du même exemplaire. Les pages intérieures du guide avaient été vendues à Louis Hachette qui avait fait relier les volumes au nom de «L. Hachette et Cie». 


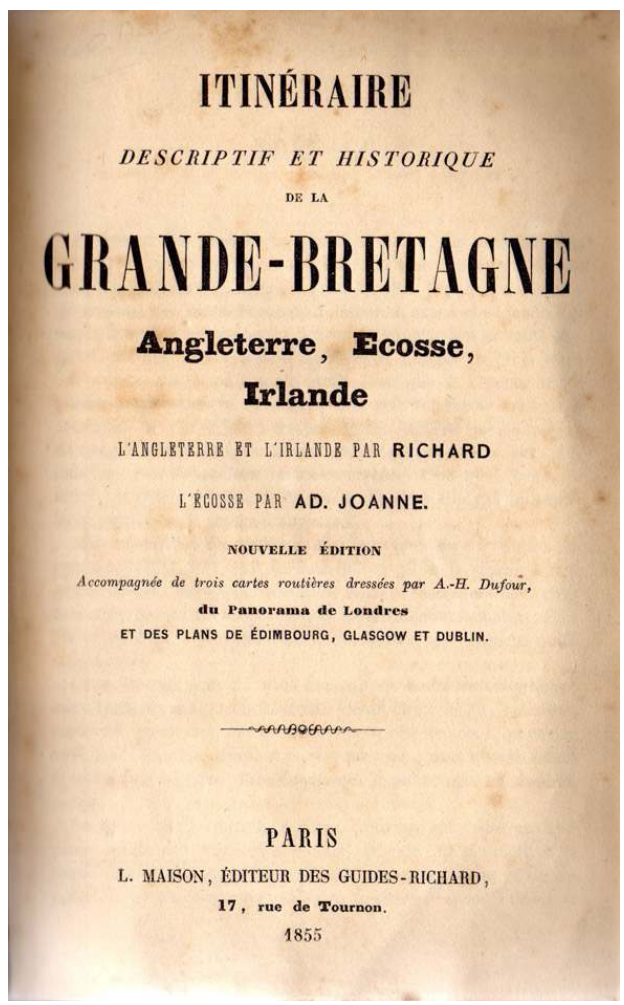

Guide Richard. Itinéraire descriptif et historique de la Grande-Bretagne. Angleterre, Écosse, Irlande. Paris : L. Maison, 1855 : couverture et page de titre du même exemplaire. Les pages intérieures du guide avaient été vendues à Louis Hachette qui avait fait relier les volumes au nom de « L. Hachette et Cie».

Une cinquantaine de titres supplémentaires enrichissent ensuite la collection naissante ${ }^{11}$. Le rachat des Guides Maison fixe aussi de manière définitive le cadre géographique des Guides de la "Bibliothèque des Chemins de fer " puis des Guides Joanne publiés par Hachette jusqu'en 1919 (fig. n³) : les destinations sont européennes avec quelques écarts en Orient (Empire ottoman et colonies françaises d'Afrique du Nord). Contrairement à Baedeker et Murray, la collection ne comprendra jamais de guides de l'Extrême-Orient, de l'Amérique du Nord ou des Indes. 


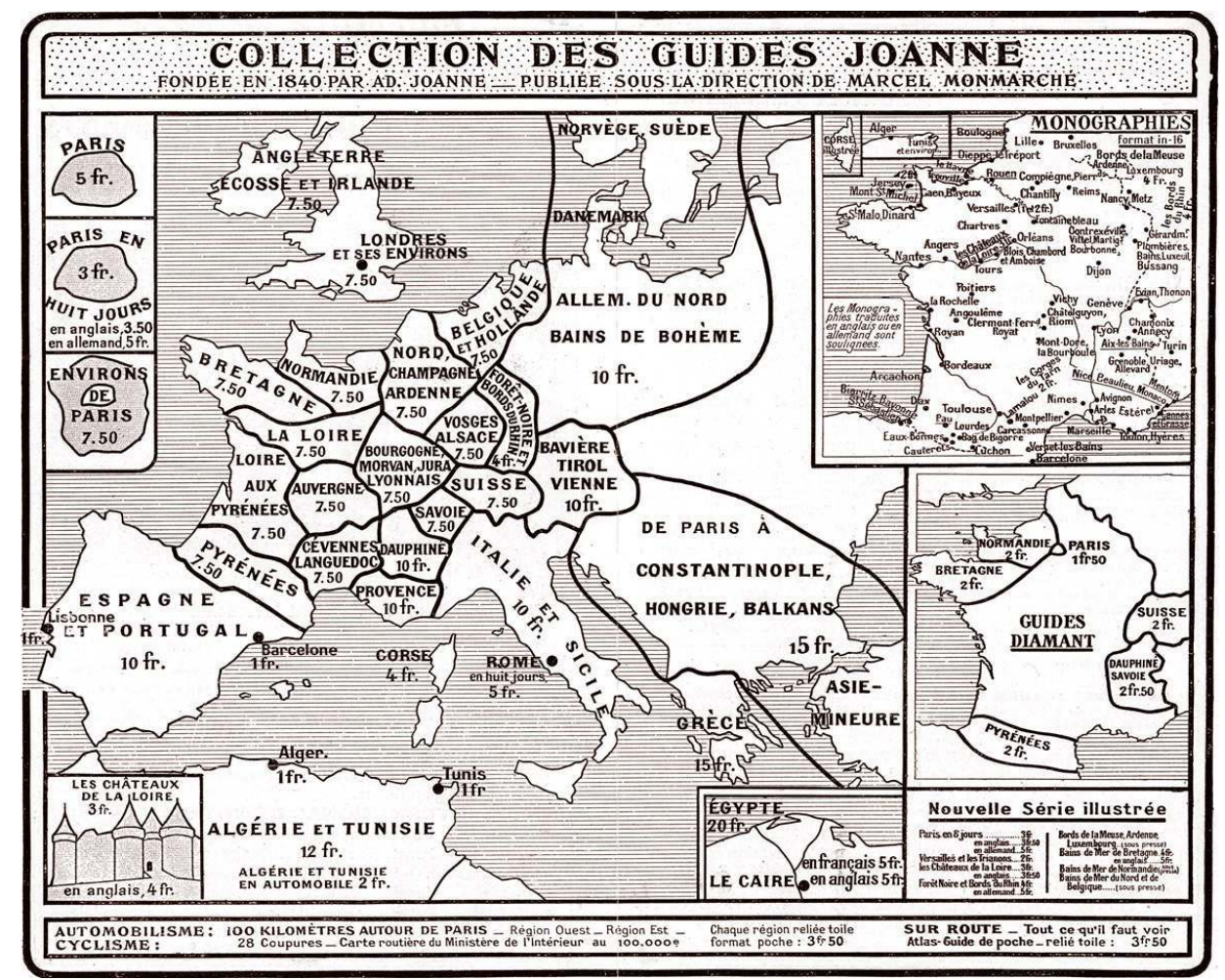

Cette carte publicitaire publiée en 1912 montre le cadre géographique de l'ensemble des collections des Guides Joanne.

$7 \quad$ Louis Hachette engage le $1^{\mathrm{er}}$ octobre 1855 le principal collaborateur de l'éditeur Maison pour en faire le directeur de sa collection de guides de voyage promise à un bel avenir. Adolphe Joanne s'était déjà fait connaître grâce à son guide de la Suisse ${ }^{12}$ publié chez Paulin en 1841 (fig. $\left.\mathbf{n}^{\circ} 4\right)$. Joanne avait passé successivement sept étés à parcourir la Suisse à pied, avec pour compagnons un guide rédigé en allemand par Ebel ${ }^{13}$ et un autre publié en anglais par Murray ${ }^{14}$. Grâce à Adolphe Joanne, un guide très complet de la Suisse est offert aux voyageurs francophones dès 1841 . Cet ouvrage novateur reprend l'exactitude des renseignements fournis par Ebel alliés à la clarté de l'organisation des itinéraires et des rubriques inspirés par Murray. Joanne revendiquait leur héritage dans l'introduction des guides rédigés par la suite : l'Écosse et l'Allemagne. 


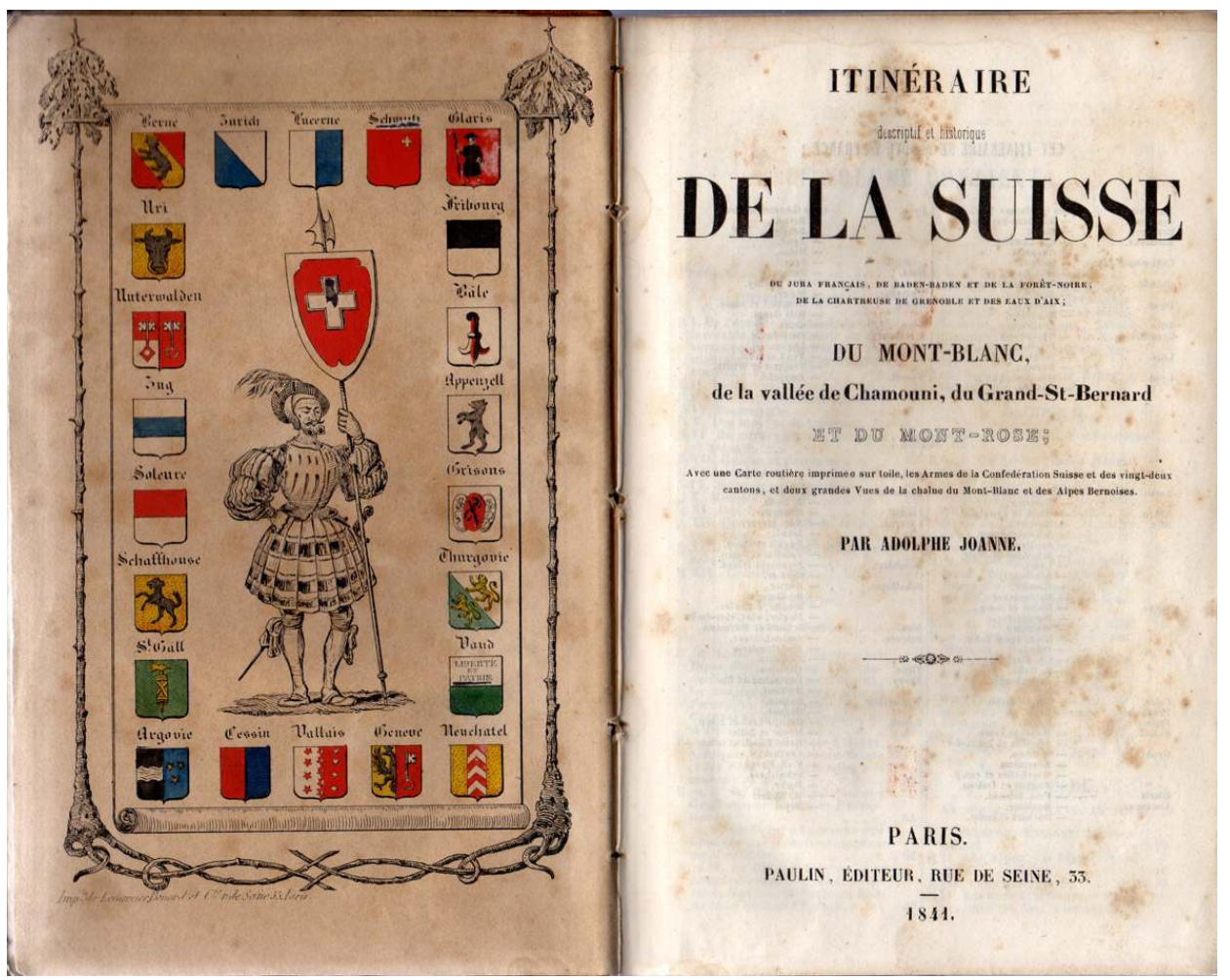

JOANNE, Adolphe. Itinéraire descriptif et historique de la Suisse [...]. Paris : Paulin, 1841. II s'agit du premier guide publié par A. Joanne.

\section{Adolphe Joanne (1855-1881) : les guides pour le voyageur} ensemble disparate issu du rachat des fonds des éditeurs concurrents (Ernest Bourdin et Louis Maison) et des créations Hachette de la première série de la Bibliothèque des Chemins de fer ${ }^{15}$. Il dirige initialement une équipe d'hommes de lettres, de traducteurs ou de voyageurs pour qui l'écriture de guides de voyage semble être une activité annexe. Il est chargé de la mise à jour de guides devenus obsolètes, de la refonte de textes qui ne correspondaient plus aux critères et de la rédaction de nouveaux titres.

Plusieurs écueils devaient en effet être évités. La confusion entre les récits de voyages et les guides était encore fréquente, comme le montre le catalogue de la Bibliothèque des Chemins de fer, qui mélange ces deux genres pourtant bien distincts. Il a aussi fallu imposer les règles rédactionnelles à des auteurs célèbres comme Jules Janin ${ }^{16}$ ou Frédéric Bernard ${ }^{17}$ et Moléri ${ }^{18}$ qui étaient déjà les auteurs de plusieurs itinéraires. Cette tâche de normalisation a été sans nul doute malaisée et le directeur de la collection dut certainement user de trésors de diplomatie ! La lecture attentive des catalogues montre que certaines collaborations prennent fin et que c'est à Adolphe Joanne que la rédaction du texte de remplacement est commandée par contrat. 
11 Les Guides de la Bibliothèque des Chemins de fer peuvent être répartis selon les deux catégories suivantes de guides « itinéraires » et de guides « cicérones ».

12 Les guides itinéraires décrivent une ligne de chemin de fer depuis Paris, voire d'une très grande ville (Bordeaux, Lyon) jusqu'à son terminus. Toutes les lignes ne font pas l'objet d'un guide : seules celles qui acheminent vers une destination touristique (Le Havre et la Normandie) ou importante (Bruxelles, Strasbourg) sont sélectionnées. Un guide était publié pour la ligne principale d'une compagnie avec laquelle un contrat de diffusion avait été signé (fig. $n^{\circ} 5, n^{\circ} 6, n^{\circ} 7$ ).

Figure 5

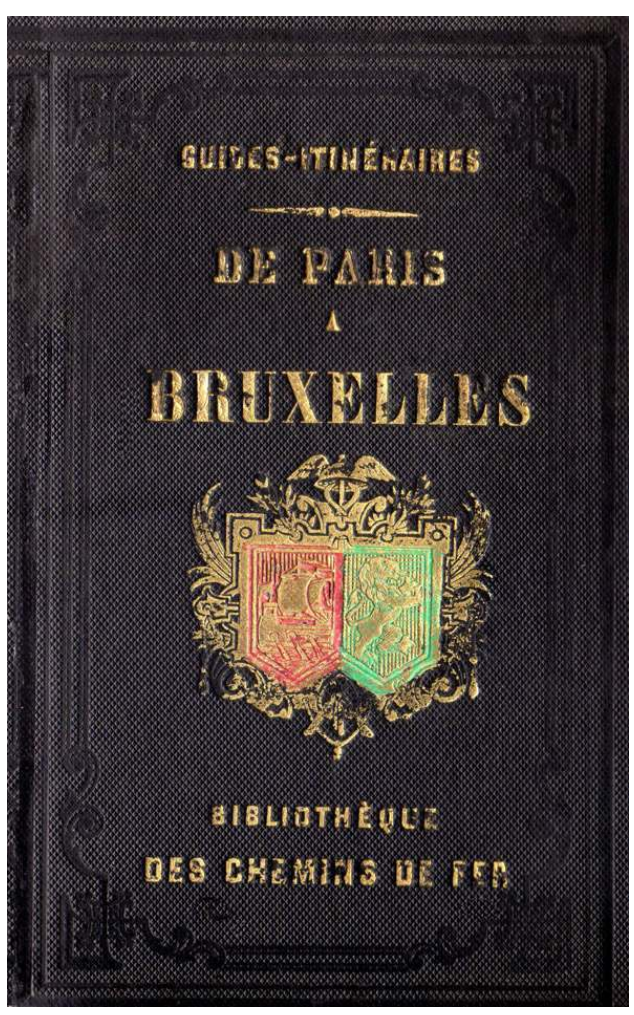

GUINOT, Eugène. Itinéraire du chemin de fer de Paris à Bruxelles, comprenant l'embranchement de Creil à St-Quentin, Ouvrage illustré de 70 vignettes dessinées par Daubigny, de cartes et de plans. Paris : Hachette, 1853, 173 p., 1 carte, 4 plans, 70 gravures (Bibliothèque des Chemins de fer ; GuidesItinéraires). 


\section{Figure 6}

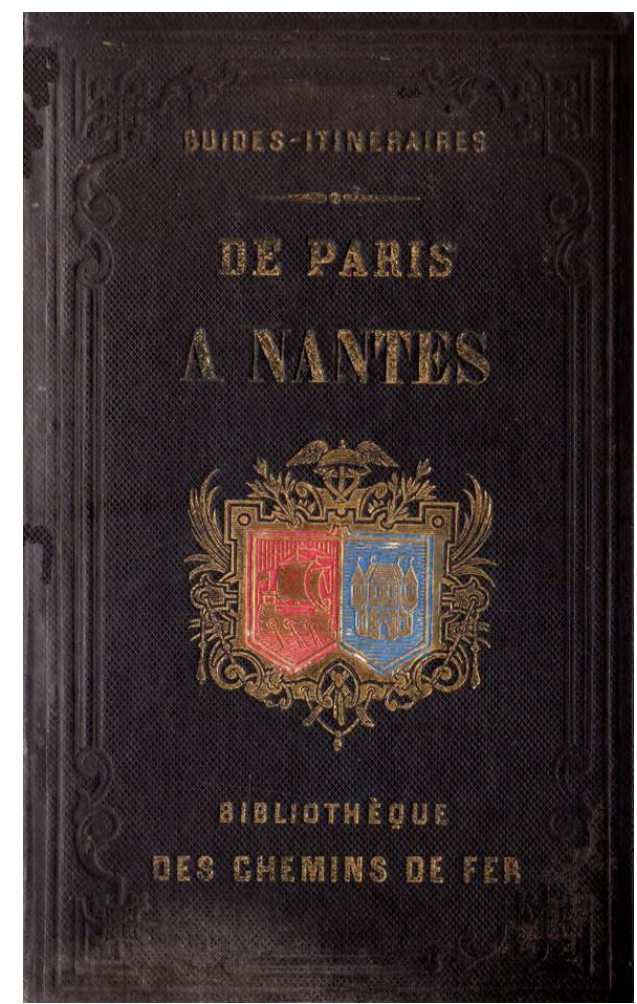

MOLERI, ACHARD Amédée, BERNARD, Frédéric. Paris à Nantes (De), $1^{\circ}$ de Paris à Corbeil et à Orléans, $2^{\circ}$ d'Orléans à Tours, $3^{\circ}$ et de Tours à Nantes, Ouvrage illustré de 110 vignettes et accompagné de 3 cartes. Paris : Hachette, 1854, XII-328 p., 3 cartes, gravures (Bibliothèque des Chemins de fer, GuidesItinéraires). 
Figure 7

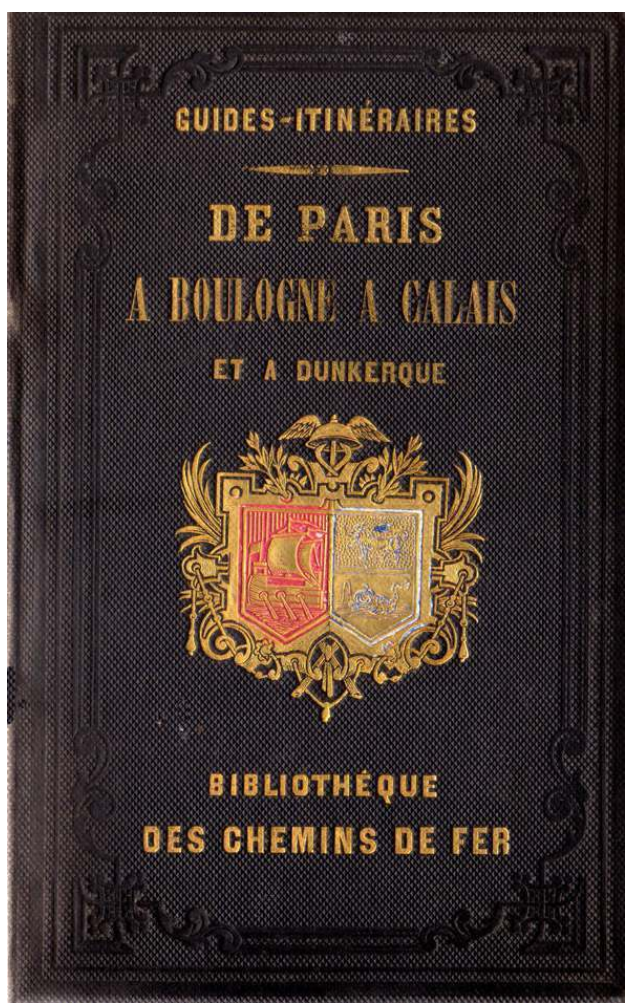

GUINOT, Eugène. Itinéraire du chemin de fer de Paris à Boulogne, à Calais et à Dunkerque, Ouvrage illustré de 80 vignettes dessinées par Daubigny, Foulquier, etc., et accompagné d'une carte. Paris: Hachette, 1855, XII-168 p., 1 carte, 80 gravures (Bibliothèque des Chemins de fer, Guides-Itinéraires).

Les Guides Cicérone regroupent plusieurs genres : les guides de villes (Paris, Londres) (fig. $\mathbf{n}^{\circ} \mathbf{8}$ ), de stations balnéaires (Dieppe, Biarritz) ou thermales (Vichy, Plombières, le MontDore), de lieux de visite plus «touristiques » mais à forte connotation aristocratique (Versailles, Fontainebleau, Chantilly, Compiègne) et enfin des guides sur un pays ou une région (Belgique, Bords du Rhin) ${ }^{19}$. 
Figure 8

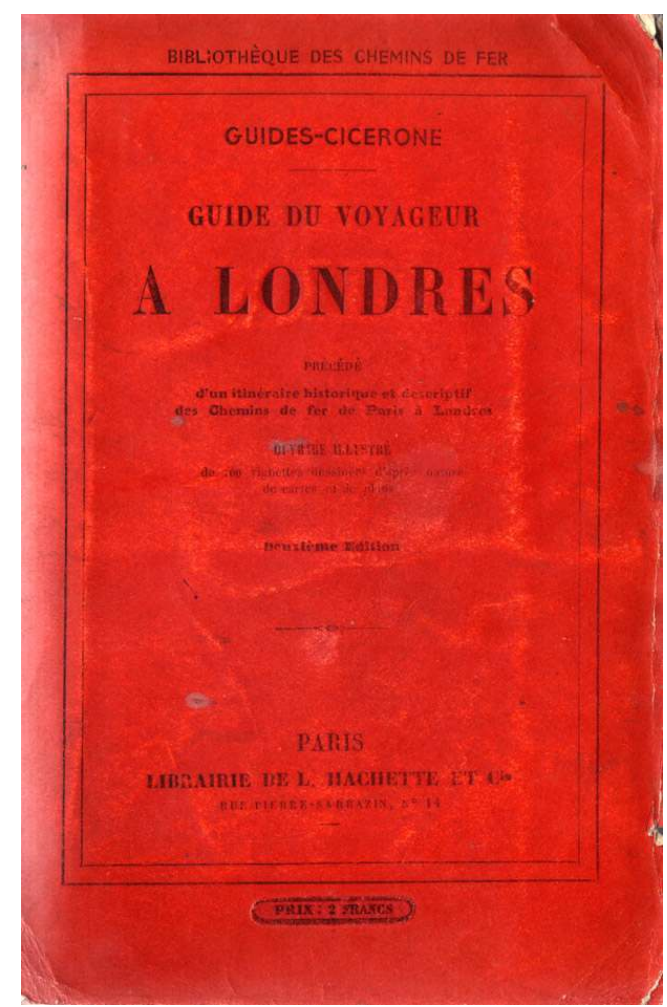

Un exemple de Guide-Cicérone : Guide du voyageur à Londres et aux environs, précédé d'un itinéraire historique et descriptif des chemins de fer de Paris à Londres. Paris : Hachette, s.d. [c. 1853], 239 p., 2 cartes, 9 plans (Bibliothèque des Chemins de fer ; Guides-Cicérone).

14 La relation entre l'ouverture d'une ligne de chemin de fer et la parution d'un guide semble assez évidente. En revanche, le sujet d'autres titres est plus surprenant. Par exemple, alors que peu de guides traitent des villes de France, un ouvrage est commandé à Jean Justin Lobet en $1855^{20}$ : le « Guide du promeneur au bois de Boulogne » qui paraît en 1856 sous le titre Le nouveau bois de Boulogne et ses alentours. (fig. $\mathbf{n}^{\circ} \mathbf{9}$ ) 
Figure 9

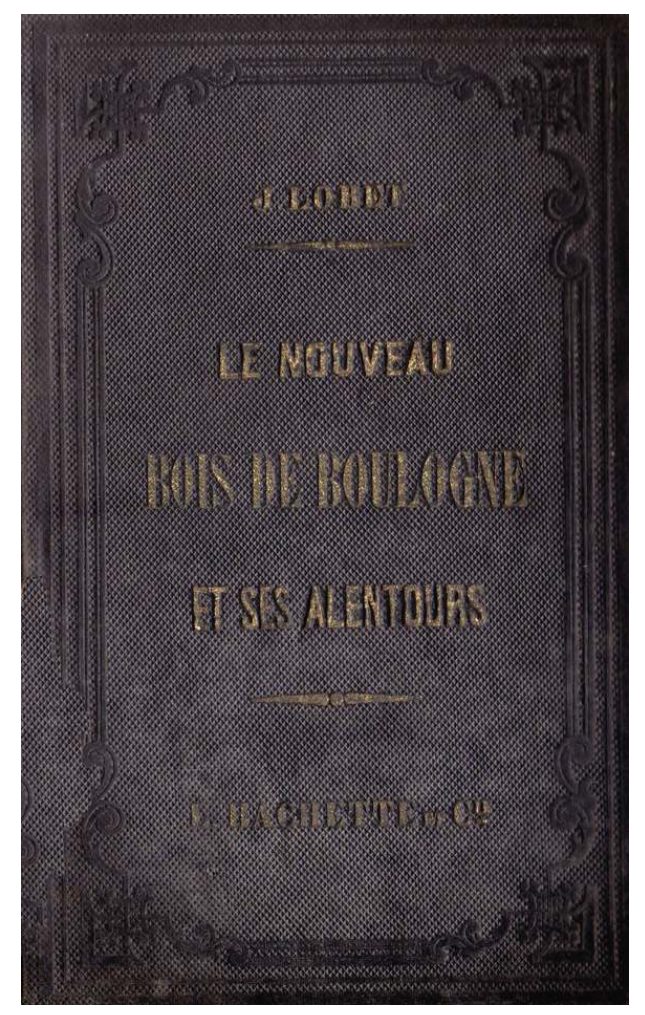

Un guide édité au moment de l'aménagement du Bois : LOBET, J. Nouveau bois de Boulogne et ses alentours... (Le), Histoire, description et souvenirs, Ouvrage illustré de 26 vignettes par Thérond. Paris : Hachette, 1856, II-156 p., 26 gravures (Bibliothèque des Chemins de fer).

L'auteur fait l'apologie du programme édilitaire derrière lequel se tiennent de grands noms proches du gouvernement comme le comte de Morny, membre du Jockey Club et instigateur de la création de l'hippodrome de Longchamp ${ }^{21}$. L'apparente étroitesse du sujet est inversement proportionnelle à l'importance des hommes politiques et spéculateurs commanditaires. De même, les louanges adressées au maire de la ville de Dieppe ou au responsable de la ligne de chemin de fer de Paris à Dieppe sont tellement appuyées $^{22}$ qu'elles ont bien du mal à dissimuler des liens et des enjeux qui dépassent largement la publication du guide : il fallait en effet rentabiliser les sommes investies pour construire la ligne de chemin de fer et les équipements de la station balnéaire ou thermale (casino, établissement de bains, hôtels) et donc attirer la clientèle. Ainsi, derrière des guides qui ont l'apparente vocation de rendre service au voyageur ou au villégiateur, se cache souvent un enjeu économique beaucoup plus vaste, dans lequel de grandes banques avaient largement investi et où Louis Hachette était souvent engagé en qualité d'actionnaire. Leur étroite relation avec l'actualité du développement politique, économique, industriel et immobilier de la France pourrait faire qualifier ces ouvrages de " guides de complaisance » publiés en remerciement de services rendus ou de valorisation d'une entreprise financière dans laquelle Hachette et ses partenaires étaient impliqués. Autre exemple, un Itinéraire de la Savoie, du Dauphiné et des Alpes françaises est commandé à Adolphe Joanne à la fin de $1858^{23}$. L'annexion de la province par la France en 1860 accélère la parution d'un tome uniquement consacré à celle-ci, le reste du contenu initialement prévu paraît séparément en deux tomes en 1862 et 1863. Le Guide Savoie est publié sans date en page de titre mais avec une préface datée de 1860 . Un compte rendu d'Albert 
Montémont s'évertuait pourtant à démentir le lien entre les deux événements ${ }^{24}$ : cependant un guide peut aussi être un vecteur de la propagande gouvernementale.

Lorsque la Bibliothèque des Chemins de fer disparaît en 1857, les guides de voyage prennent le nom de "Guides-Joanne », ce qui semble s'imposer naturellement, mais ne signifie nullement l'abandon des titres déjà parus. Comme cela s'était passé précédemment, les guides sont déplacés d'une collection à une autre : les guides rachetés à Bourdin et Maison avaient été reliés avec la couverture Hachette, ceux de la défunte Bibliothèque des Chemins de fer sont habillés de la couverture des Guides Joanne avec parfois une adaptation de la page de titre. Ce procédé de " glissement » d'une série à une autre est utilisé tout au long de l'histoire des guides publiés par Hachette, entreprise pour laquelle la rentabilité était un maître mot.

\section{L’« Itinéraire général de la France »}

17 À partir de 1860, Adolphe Joanne dirige la structuration de la collection et crée des séries cohérentes comme l'«Itinéraire général de la France» (appelé ensuite IGF) et l'« Itinéraire de l'Orient ».

18 La mise à jour des guides ferroviaires décrivant un itinéraire de ligne de chemin de fer est remplacée par une description complète de la région à visiter ; l'organisation des guides par compagnies de chemins de fer subsiste sous le titre de Guide du voyageur en France publié sous le nom de Richard, jusqu'en 1892, en 5 volumes vendus séparément. De même, des brochures sont éditées à bas prix sans doute pour maintenir la concurrence avec Chaix. Toutefois, c'est l'IGF qui, par l'ampleur de l'entreprise, devient prépondérant.

La couverture de l'ensemble de la France n'est pas planifiée d'emblée ; le schéma éditorial se construit au fur et à mesure. Prévu en dix volumes en 1862 (8 pour la France et 2 pour Paris et ses environs), l'IGF regroupe jusqu'à une vingtaine de guides (fig. $\mathbf{n}^{\circ} \mathbf{1 0}$ ). 


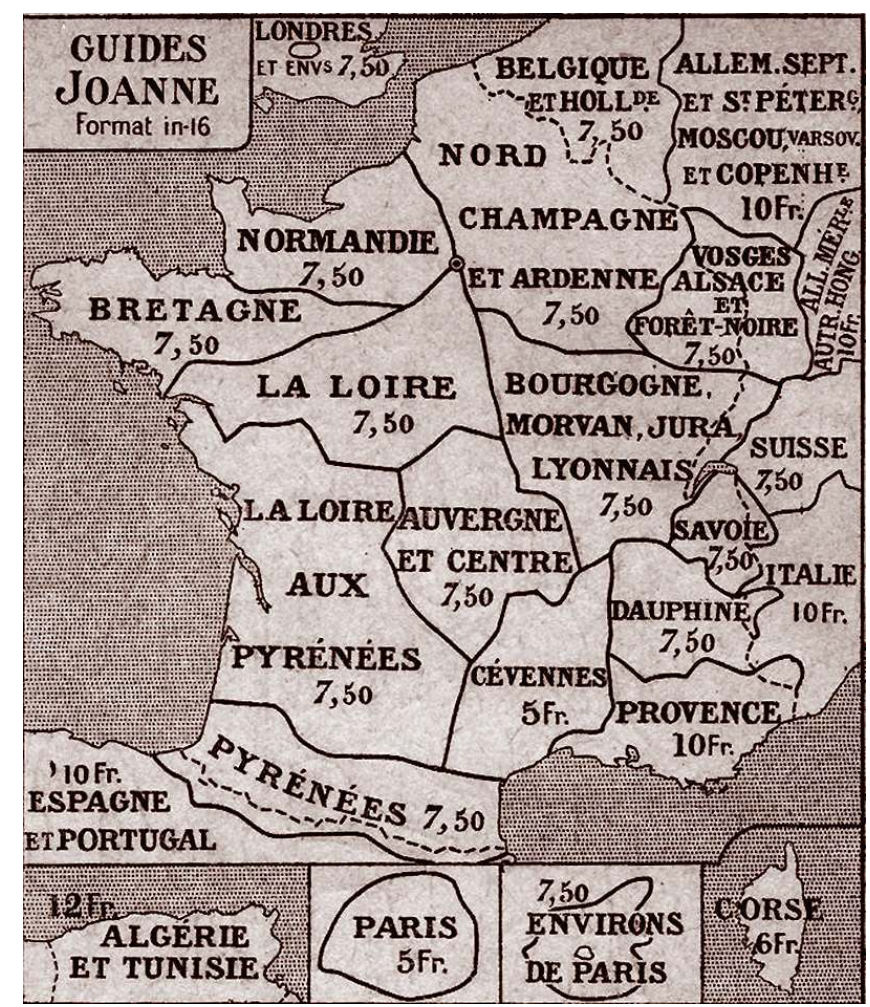

Itinéraire général de la France : cette carte montre le découpage en 13 volumes de la France auxquels il faut ajouter deux volumes pour Paris et ses environs soit un total de 15 volumes (Cévennes, 1908).

Dès son arrivée chez Hachette, Adolphe Joanne signe un contrat de commande d'un Itinéraire descriptif et historique de la France prévu en deux volumes : I, Centre et Midi et II, Est et Ouest ${ }^{25}$. Trois ans plus tard, alors que ce guide n'avait pas vu le jour, un nouveau contrat est établi pour un Dictionnaire universel des communes de France ${ }^{26}$. Les recherches et la rédaction pouvaient être menées de front à condition de répartir la documentation en volumes séparés. Il était aussi possible de reprendre des informations utiles pour chacun des ouvrages. Les premiers guides font partie de la ligne de chemin de fer du Paris-LyonMarseille (PLM) et portent en sous-titre les noms des régions traitées. Entre 1862 et 1868, la couverture de la France est menée à bien dans la collection des Guides Joanne pendant que le dictionnaire parait en 1864 et une version corrigée réimprimée en 1869.

Les Guides de l'IGF présentent plusieurs innovations intéressantes dont la cartographie est la plus évidente. Déjà, aux cartes des lignes de chemin de fer héritées des Guides Maison avaient été substituées des cartes systématiquement orientées nord-sud, important progrès vers la lisibilité et l'appréhension logique du voyage et du territoire. Elles sont ensuite complétées par des cartes de chacun des départements concernés par le guide ainsi que de cartes de détails des côtes ou des massifs. Sur les panoramas des massifs montagneux sont portés les hauteurs des sommets et leurs noms, reflet de l'avancée des connaissances géographiques de l'époque (fig. $\left.\mathbf{n}^{\circ} \mathbf{1 1}\right)$. Les plans de villes sont aussi de plus en plus nombreux. 


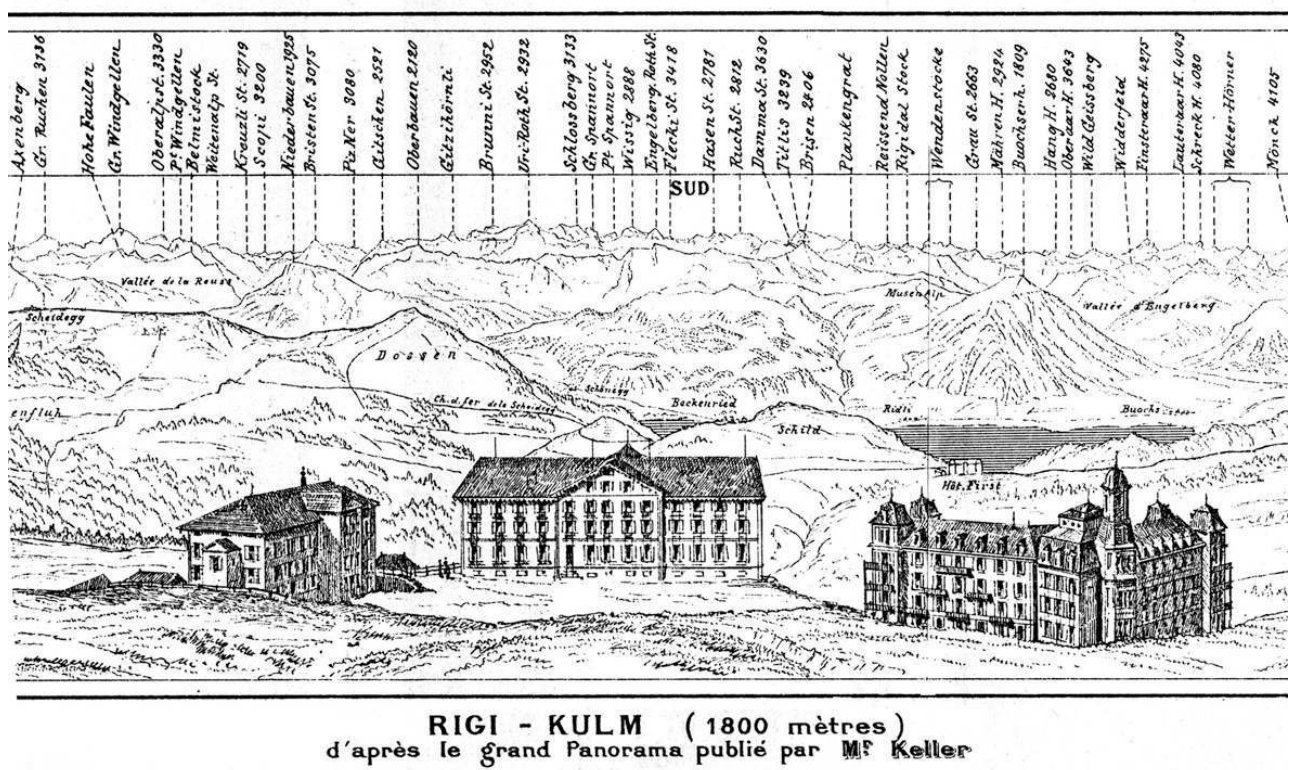

Panorama dépliant du Rigi-Kulm avec identification des sommets et mentions de leurs altitudes (Suisse, 1909).

La structuration des données selon un plan établi pour l'ensemble des volumes permet à quiconque de trouver facilement les informations recherchées qu'elles soient d'ordre touristique ou général (histoire ou production économique d'une région ou d'une ville). Les principales «curiosités touristiques » sont mises en valeur par des gravures qui les représentent. Cet intérêt pour les richesses, construites ou naturelles, peut être mis en relation avec les études des savants de l'époque. En effet, sous le Second Empire, s'épanouissent les travaux de Mérimée ou de Viollet-le-Duc dans le domaine du patrimoine historique et archéologique (fig. $\left.\mathbf{n}^{\circ} \mathbf{1 2}\right)$. 


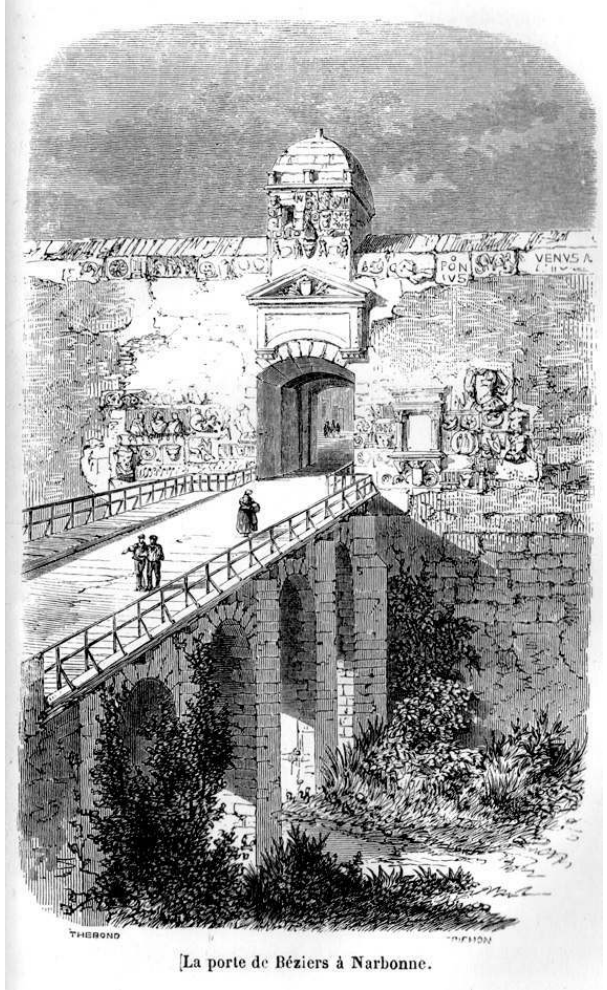

Des gravures illustraient les premiers Guides de la Bibliothèque des Chemins de fer et les Guides Joanne. Elles disparaissent peu à peu des Guides Itinéraires trop volumineux pour compléter les Guides Diamant puis les Monographies (De Bordeaux à Toulouse, à Cette et à Perpignan, 1858).

Cette couverture uniformisée du territoire en dix volumes traduit un désir d'unification et de normalisation de l'ensemble de la France, dont jusqu'ici seules quelques régions pittoresques (Pyrénées, Savoie) avaient fait l'objet d'un guide. Ces guides rédigés en français - langue du visiteur mais pas forcément des autochtones - utilisent des unités de mesure métriques et une seule monnaie (le franc) dans un pays où il était encore très courant de compter dans des monnaies de l'Ancien Régime (on parlait encore de «milliers de livres de rente» dans les romans de la fin du XIX ${ }^{e}$ siècle pour situer la richesse d'un personnage), d'arpenter la terre et de mesurer sa production selon des unités variables d'une province à l'autre ${ }^{27}$. De même, la cartographie utilise l'échelle du département, manière efficace de diffuser un type de découpage territorial, dont l'utilité pour le voyageur reste contestable, mais pouvait en revanche être montrée aux hôtes du voyageur. Ces cartes départementales polychromes sont simultanément vendues séparément ; elles illustraient aussi les fascicules des Géographies départementales publiées par Hachette sous la direction d'Adolphe puis de Paul Joanne. Le dessin et la gravure des cartes départementales étaient ainsi parfaitement rentabilisés par l'éditeur.

L'enseignement avait toujours été un des buts de Louis Hachette qui en avait fait sa devise 28. Il semble que ces guides répondent à cette volonté de transmettre des connaissances par tous les moyens. Ceci semble en grande partie avoir été atteint car de nombreuses et prestigieuses bibliothèques, comme la Biblioteca Marciana de Venise, font l'acquisition de l'ensemble des dix volumes de l'IGF dès leur parution, sans acheter les mises à jour parues par la suite : on devait considérer ces guides comme une somme encyclopédique résumée qui ne servait pas nécessairement pour un voyage mais jouait un rôle documentaire. 
25 La France était par ailleurs très peu traitée par les grandes collections concurrentes : 2 volumes par Murray, 3 puis 4 par Baedeker ${ }^{29}$ : le champ d'étude était libre et la Librairie Hachette a su s'en emparer. La couverture du Royaume Uni est entreprise à peu près à la même époque par les Guides Murray $^{30}$ mais, semble-t-il, plus lentement et sans la même systématique foudroyante. En revanche, il faut attendre les revers subis par l'Allemagne après la première guerre mondiale pour que les Guides Baedeker proposent aux lecteurs allemands des guides détaillés de leur pays, province par province ${ }^{31}$.

Deux guides publiés chez Maison ${ }^{32}$ précèdent L'Itinéraire de l'Algérie, pays alors inclus dans les départements français, qui était rattaché à la série de l'IGF. La cartographie fréquemment mise à jour ainsi que les nouveaux chapitres consacrés aux régions récemment "pacifiées", donc visitables pour le touriste, sont une bonne vitrine de l'avancée coloniale de la France en Afrique du Nord.

\section{L'Itinéraire de l'Orient, des guides pour spécialistes ?} consacrée à l'Orient, déclinée en plusieurs volumes. Un Guide de l'Orient figurait dans le fonds racheté à Louis Maison. CEuvre de Richard et Quétin, publié plusieurs fois, cette dernière édition, parue en 1855 , nécessitait une refonte selon les nouveaux critères établis par Adolphe Joanne. En 1858, il cosigne avec Émile Isambert un contrat pour la rédaction d'un nouvel Itinéraire de l'Orient ${ }^{33}$. Ce nouveau volume fait appel à de nombreux contributeurs (géographes, linguistes, archéologues, etc.) dont les recherches scientifiques sont mises à profit pour faire de ce guide un bilan des connaissances de l'époque, méthode déjà observée pour l'IGF. Cet itinéraire paraît d'abord en 1861 sous la forme d'un volume unique, regroupant l'ensemble des territoires de l'Empire ottoman (à l'exception de la Libye), de la Grèce et des Balkans. Un aspect frappant est l'appel à la contribution des voyageurs qui sont courtoisement mais fermement invités à collecter le maximum d'informations sur les régions qu'ils parcouraient. Ces régions difficiles d'accès étaient souvent le but du voyage d'une vie, les moyens financiers et humains étant extrêmement lourds. Toutefois, les sollicitations des auteurs dépassaient très largement les compétences d'un voyageur amateur d'orientalisme. En effet, il convenait de se munir d'appareils de mesure scientifique pour faire des relevés géographiques ou d'apprendre à faire des moulages d'inscriptions antiques et de les rapporter en France, ainsi que de noter tout ce qui pouvait servir au développement des connaissances sur l'Orient en France.

L'édition suivante est une somme séparée en trois volumes (1873-1878-1882), dont la partie la plus spectaculaire concerne un voyage d'exploration en Abyssinie. En effet, la lutte pour la découverte des sources du Nil faisait alors rage et la France était engagée dans cette course de vitesse face à l'Angleterre. La cartographie très aboutie fait la synthèse des connaissances historiques et géographiques. Elle est réunie pour la Terre Sainte dans un portefeuille de cartes séparées en plusieurs feuilles ce qui donne une idée de l'importance accordée à cet aspect de la documentation (fig. $\mathbf{n}^{\circ} \mathbf{1 3}, \mathbf{n}^{\circ} \mathbf{1 4}$ ). À cette époque, les Européens étaient engagés dans un processus de colonisation qui avançait parfois de façon masquée. Sans aller jusqu'à l'annexion de contrées entières, une zone d'influence culturelle puis économique pouvait être mise en place comme cela avait été le cas après l'Expédition d'Égypte. Le voyageur érudit n'est-il pas un excellent ambassadeur 
de sa culture et un bon entremetteur entre les élites afin d'établir des relations plus étroites?

\section{Figure 13}

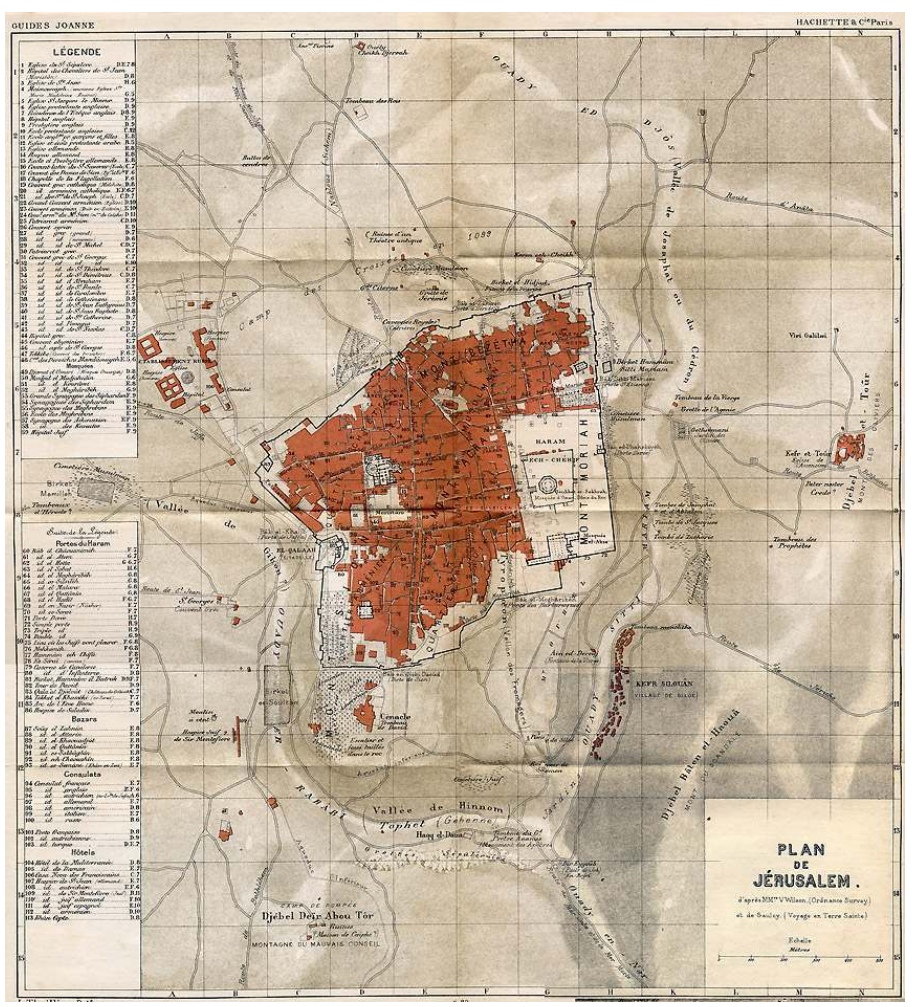

Plan de Jérusalem, extrait du portfolio de cartes correspondant à l'édition de 1882 contenant les cartes et plans suivants : Palestine et Liban, Arabie Pétrée, Environs de Jérusalem, Sinaï ; plan de Jérusalem. II semble que la carte de Syrie du Nord ait été ajoutée après 1890. 


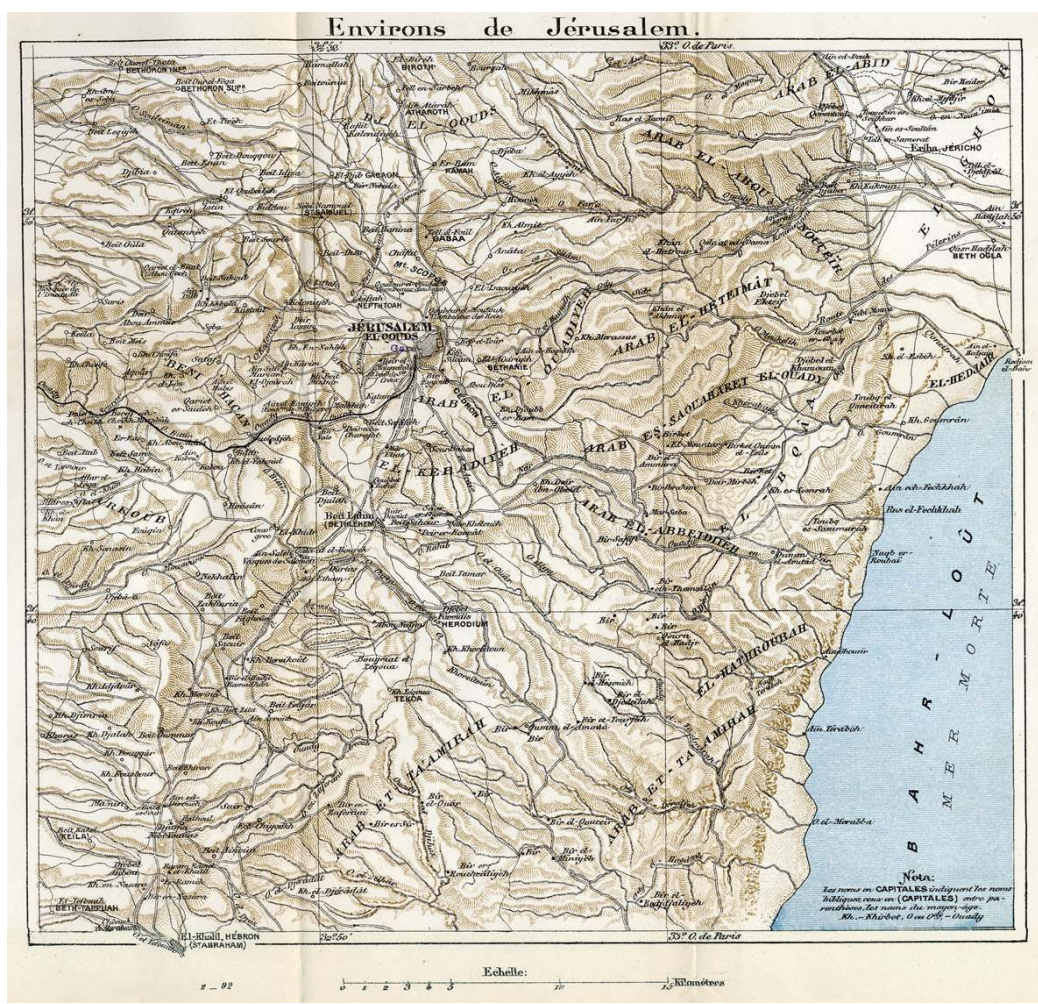

Carte des environs de Jérusalem, extrait du portfolio de cartes correspondant à l'édition de 1882 contenant les cartes et plans suivants : Palestine et Liban, Arabie Pétrée, Environs de Jérusalem, Sinaï ; plan de Jérusalem. II semble que la carte de Syrie du Nord ait été ajoutée après 1890.

\section{Les Guides Diamant : une première série destinée au touriste}

En 1866, la création de la sous-collection des Guides Diamant répond à un type de voyage en développement: celui du touriste plus pressé, moins érudit mais toutefois assez exigeant. Cette collection s'adapte aux désirs d'une nouvelle clientèle par son format plus petit, son papier bible et les sujets traités. Le premier titre de la série est Paris, publié en 1866 pour l'Exposition universelle de 1867 ; la même édition paraît en plusieurs langues, événement qui n'est pas répété pour les autres titres. Le second titre édité la même année est la Suisse, dont l'espace avait été conquis et banalisé par des touristes bien différents des premiers géographes alpinistes qui en exploraient les cimes. 
Figure 15

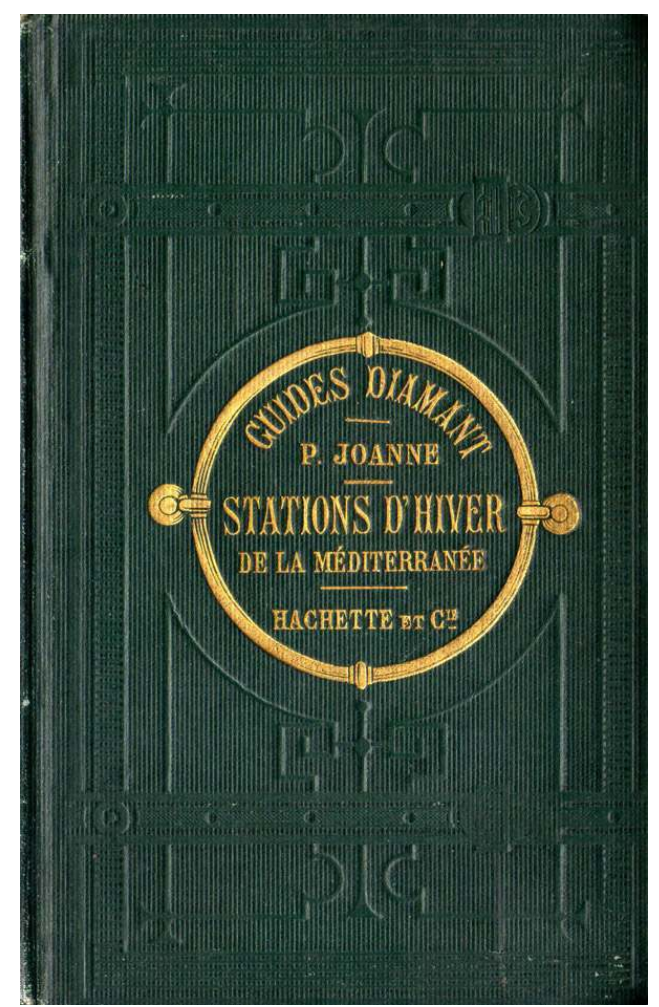

Guide Diamant de la Côte d'Azur avant qu'elle ne soit nommée ainsi : Les stations d'hiver de la Méditerranée (3e édition, 1879) par Paul Joanne qui remplace le Guide des Villes d'hiver de la Méditerranée et les Alpes maritimes, par Élisée Reclus (1864)

Ces guides sont une présentation abrégée du contenu des Guides Itinéraires Joanne, traitant des destinations faciles d'accès grâce au chemin de fer ou aux steamers :

- régions françaises déjà très fréquentées (Bretagne, Dauphiné et Savoie, Normandie, Pyrénées, la côte méditerranéenne, (fig. n¹5) - actuelle Côte d'Azur - et les Vosges) ;

- Paris et des villes importantes situées sur le parcours de la ligne PLM (Paris, Lyon, Marseille) ou desservant des stations balnéaires (Bordeaux avec Royan, Soulac et Arcachon);

- lieux de villégiatures balnéaires et de cures thermales nouvellement créés ;

- capitales européennes (Londres, Rome) et pays limitrophes de la France (Belgique, Hollande, Autriche-Hongrie, Allemagne du Sud, Espagne et Portugal, Italie, Suisse). Un guide de circonstance est édité pour l'exposition universelle de Vienne en 1873 où les industriels français dont Hachette possédaient des stands (fig. $\mathbf{n}^{\circ} \mathbf{1 6}$ ). 
Figure 16

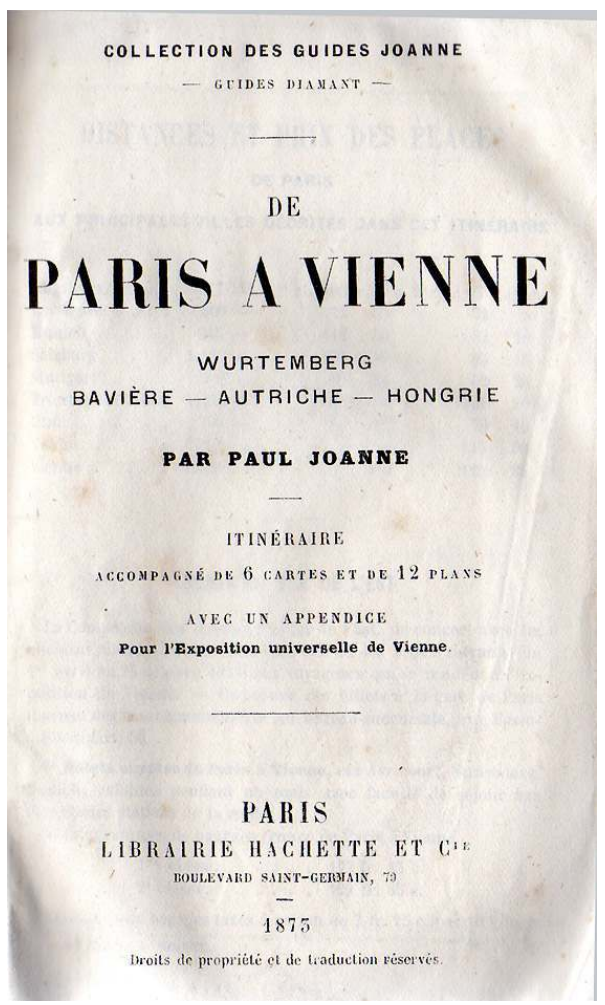

Guide Diamant De Paris à Vienne, Wurtemberg, Bavière, Autriche, Hongrie, publié à l'occasion de l'exposition universelle de Vienne (1873) ; l'appendice consacré à l'exposition est relativement bref : 7 pages mais un plan en couleurs permet de s'orienter.

Pendant le dernier quart du XIX siècle, ces guides abrégés remplacent les Guides Itinéraires pour les destinations les plus faciles d'accès (Belgique, Allemagne) ou sont publiés parallèlement aux guides plus érudits de l'Espagne, de l'Italie ou de la Suisse en plusieurs volumes. Les titres des Guides Diamant, moins onéreux que les grands Guides Itinéraires, correspondent à ceux publiés par le concurrent Baedeker, en français et au même moment.

\section{Paul Joanne (1881-1911) et Marcel Monmarché (1911-1919) : les guides de la villégiature et du tourisme automobile}

Paul travaille en collaboration directe avec son père avant de le remplacer en 1881. Voyageur et alpiniste comme lui, il connaissait les contraintes imposées à l'excursionniste et au randonneur et savait être attentif à l'évolution des modes de déplacement qui a lieu dès la fin du siècle. Sa direction des Guides Joanne est marquée par la diversification des collections et l'adaptation constante aux tendances du tourisme en plein développement, ainsi que l'augmentation sensible du nombre de nouveaux titres par an, même s'il s'agissait d'une simple mise à jour des renseignements pratiques et de quelques addenda. 


\section{Une nouveauté rentable : les monographies} disponibles dans les gares à des prix très abordables $(50$ centimes et $1 \mathrm{~F})$ qui remplacent peu à peu les Guides Diamant dont le prix variait entre 2 et $6 \mathrm{~F}$. La recette, simple et astucieuse, consistait à publier à part des extraits des Guides Itinéraires, beaucoup plus onéreux (entre 5 et $15 \mathrm{~F}$ ), extraits consacrés d'abord à des villes, puis ensuite à des lieux de villégiature. Les premières années, ces tirés à part brochés ne comportaient même pas de page de titre. Au fur et à mesure, les monographies sont enrichies de plans de la ville traitée et de cartes des environs avec un développement des excursions, ainsi que de gravures puis de photographies quand cette technique remplace la précédente.

Des titres concernent les lignes des grandes compagnies de chemin de fer, d'autres les "bains de mer", et des curiosités touristiques historiques ou naturelles (mont Saint Michel, Esterel, gorges du Tarn). Les monographies des villes étrangères (Venise, Rome) sont de simples extraits. À la faveur d'un événement particulier comme une exposition nationale ou universelle, une monographie était spécialement éditée avec un plan des lieux de la manifestation. Certains guides portent à la place du nom de la collection la mention de l'événement ce qui laisse supposer qu'il s'agit de commandes des organisateurs à l'éditeur. Plusieurs titres sont publiés en relation directe avec une exposition :

- Genève pour l'exposition de $1896^{34}$,

- Liège, Spa et ses environs pour l'exposition tenue à Liège en $1905^{35}$,

- Marseille et l'exposition coloniale en 1906 (fig. $\left.\mathbf{n}^{\circ} \mathbf{1 7}\right)^{36}$,

- Turin et ses environs pour l'exposition de $1911^{37}$,

- Berlin et Leipzig pour la manifestation de Leipzig en 1914",

- Berne, pour l'Exposition nationale suisse en $1914^{39}$. 
Figure 17

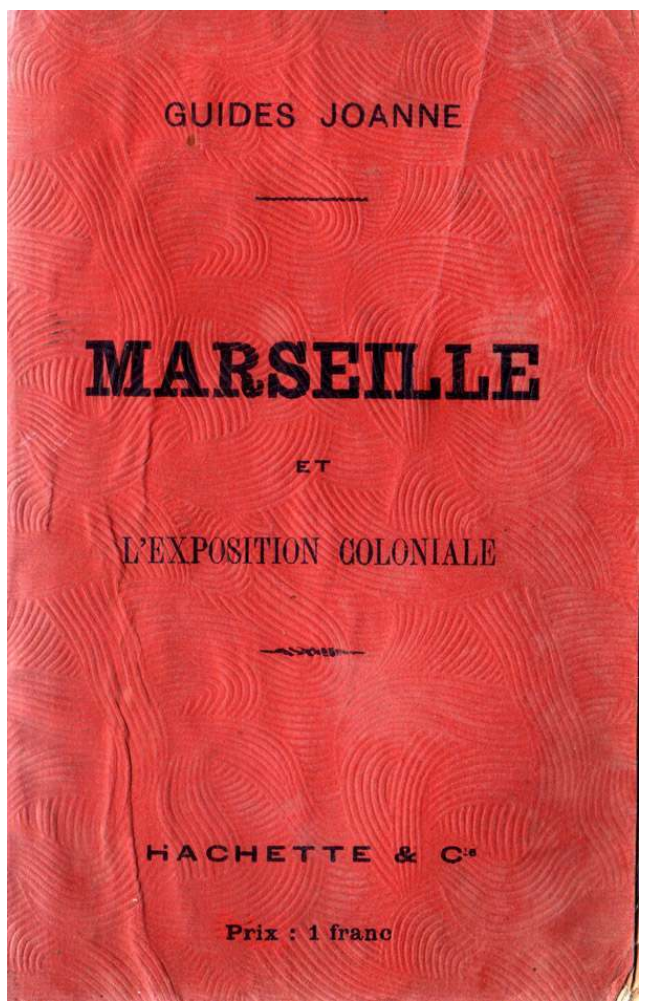

Monographie Marseille et ses environs : la couverture annonce un contenu supplémentaire ; un appendice de 3 pages avec un plan est consacré à l'exposition coloniale de 1906.

D'autres titres sont spécialement conçus pour répondre à une mode particulière comme celle des bains de mer. Deux monographies répondent directement aux attentes d'un public familial moins fortuné qui profite des billets de chemin de fer à tarif réduit dits «billets de bains de mer » : ce sont Les plages de Bretagne $e^{40}$ paru en 1900 et 1905 et Bains de mer de l'État ${ }^{41}$ (fig. $\mathbf{n}^{\circ}$ 18) publié en 1899. Ce dernier titre est manifestement un ouvrage commandé par la compagnie des chemins de fer de l'État qui dessert les plages et stations balnéaires dont le guide fait la promotion. 
Figure 18

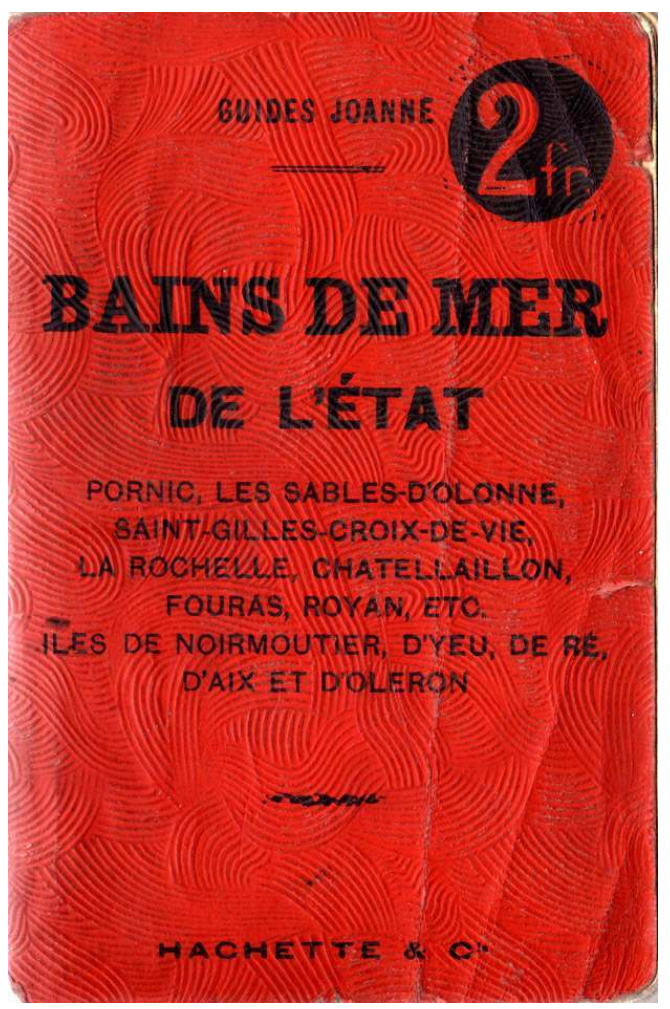

Monographie Bains de mer de l'État, Pornic, les Sables d'Olonnes, St-Gilles-Croix-de-Vie, La Rochelle, Chatellaillon, Fouras, Royan, etc., Iles de Noimoutier, d'Yeu, de Ré, d'Aix et d'Oléron : la couverture donne la liste des plages desservies par la compagnie de chemin de fer de l'État (1899).

La monographie Lisbonne a été spécialement commandée en 1912 à l'éditeur Hachette par la Librairie Ferreira de Lisbonne qui voulait pouvoir proposer à sa clientèle internationale un Guide Joanne sur sa ville ${ }^{42}$. Enfin, le titre Bosnie-Herzégovine est publié en $1897^{43}$ en relation avec l'exposition universelle tenue à Bruxelles la même année puis avec celle de Paris en 1900, où un pavillon indépendant mettait à l'honneur cette région sous administration austro-hongroise.

38 Faciles à fabriquer et à mettre à jour, ces petits guides, reflets de l'actualité événementielle, renvoient à l'image d'une Europe dans laquelle il était aisé de se déplacer pour assister à quelque manifestation importante (fig. $\left.\mathbf{n}^{\circ} \mathbf{1 9}\right)$. Ils sont aussi un bon relais pour la propagande gouvernementale de pays qui ont besoin de mettre en avant leur culture ou leur développement économique. 


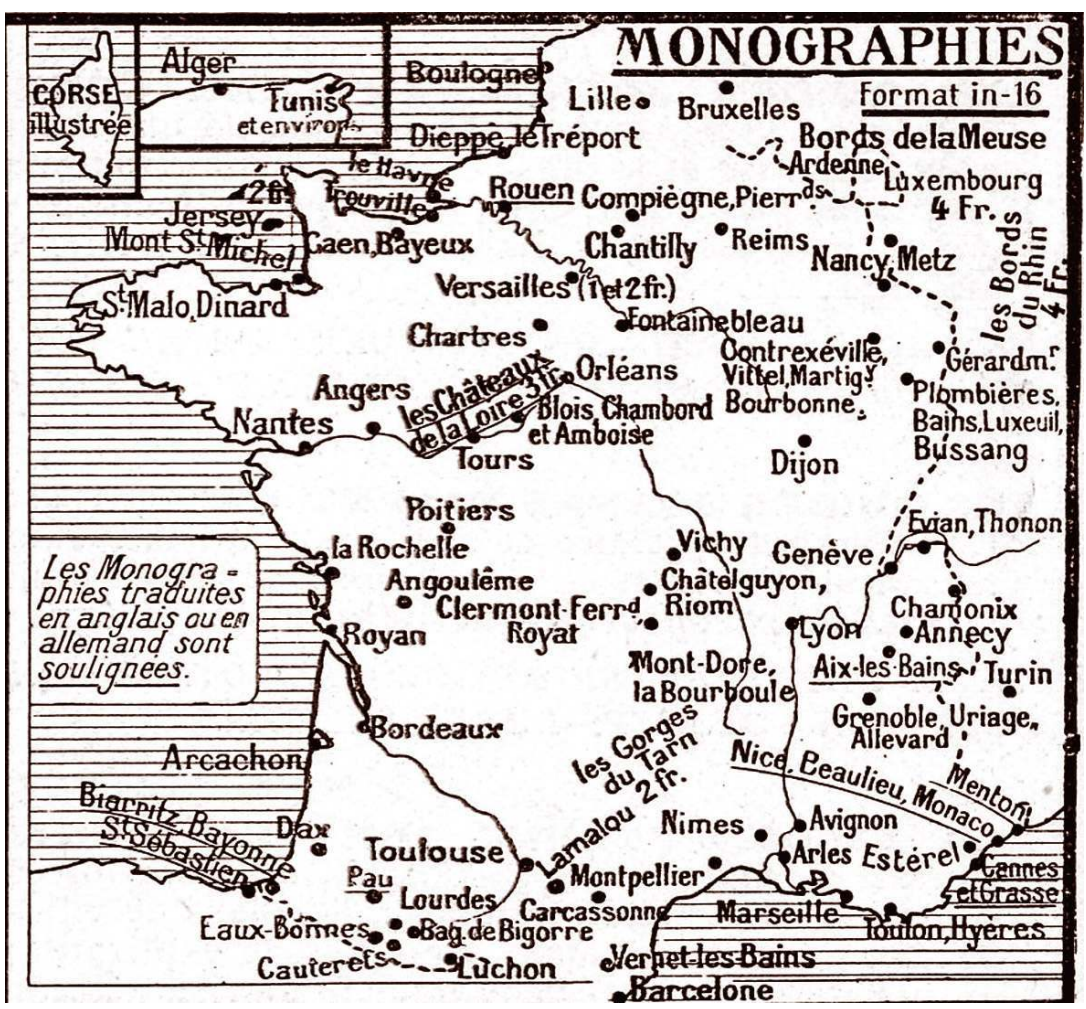

Monographies : cette carte recense les titres publiés depuis 1887 jusqu'en 1912. Certains titres sont publiés en langues étrangères (anglais, allemand et espagnol).

\section{Le devenir des séries existantes}

Parallèlement, les Guides Diamant sont réduits aux quelques titres qui font leur succès ( Paris, Bretagne, Dauphiné et Savoie, Normandie, Pyrénées, Stations d'hiver de la Méditerranée) avec une modification d'importance en 1899 : la reliure de percaline vert émeraude est abandonnée au profit d'un brochage rouge semblable à celui des monographies (sauf pour Stations d'hiver de la Méditerranée). L'impression est faite sur un papier très ordinaire et le prix baissé à $2 \mathrm{~F}$. La couverture porte surtout deux mentions importantes reprises en page de titre : la première, « Routes les plus fréquentées », montre que le guide s'adresse à des touristes qui empruntent les sentiers battus; la seconde est l'apparition d'une date après le titre, par exemple "Bretagne 1899 ", qui met en valeur une mise à jour annuelle des renseignements pratiques et rend le guide caduc l'année suivante (fig. $\left.\mathbf{n}^{\circ} \mathbf{2 0}\right)$. Cette entrée dans le monde de la consommation du guide jetable supposait de pouvoir maintenir le rythme des mises à jour. Si les modes de communication (télégraphe, téléphone) avaient fait d'immenses progrès, il fallait obtenir des réponses précises aux demandes de renseignements et cela dans les délais nécessaires pour la mise sous presse. C'est certainement l'écueil insurmontable auquel se heurtent les rédacteurs : l'expérience est arrêtée, cinq ans plus tard. En 1904, on conserve seulement la mention « Routes les plus fréquentées" annonciatrice des pratiques d'un tourisme démocratisé pour les titres suivants : Bretagne, Dauphiné-Savoie, Normandie, Pyrénées et Suisse. 


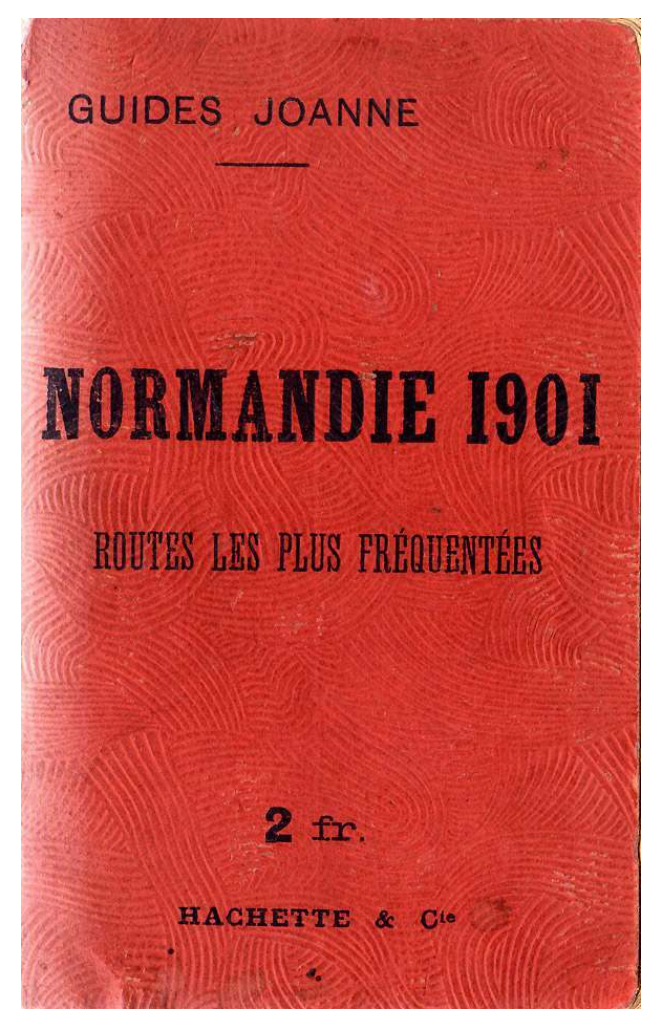

Guide Diamant Normandie 1901 : la reliure en percaline gaufrée est remplacée par un brochage rouge ordinaire.

L'IGF continue d'être développé, constamment mis à jour et adapté par la scission d'épais volumes en plusieurs tomes, de même que les guides consacrés aux destinations culturellement riches (Espagne, Italie, Grèce) ou aux curiosités naturelles abondantes (Suisse). Une nouveauté importante est le guide De Paris à Constantinople publié en relation avec l'ouverture de la ligne de l'Orient express, inaugurée en octobre $1883^{44}$. L'intérêt pour l'Europe de l'Est apparaît avec les tomes intitulés États du Danube et des Balkans ${ }^{45}$, guides qui renouent avec un genre de voyage plus aventureux. En revanche, les destinations délaissées par les touristes ne font plus l'objet de guides dont la réalisation et la mise à jour était longue et coûteuse : L'Itinéraire de l'Orient, une fois épuisé, est remplacé par un ouvrage unique : l'Égypte, destination alors très à la mode. Ce guide est réparti en trois volumes (généralités, Haute et Basse-Égypte) réunis dans un coffret, présentation astucieuse qui permettait de n'emporter que le tome nécessaire pour la visite. Ce procédé était utilisé de façon moins luxueuse pour rendre les Guides de l'IGF plus légers: diminution du format, possibilité de détacher des parties brochées à l'intérieur de la reliure, retenue ou non par des élastiques (Paris, 1892), système ingénieux qui, contrairement aux Guides Baedeker, ne détruisait pas le livre. Les Guides Itinéraires étaient desservis par leur épaisseur, leur poids et sans doute leur prix élevé ; pourtant, au cours des premières décennies du $\mathrm{XX}^{\mathrm{e}}$ siècle, ils eurent un retour en grâce alors que les Guides Diamant étaient abandonnés. 
Les guides illustrés, le villégiateur et le touriste cycliste ou automobiliste

Figure 21

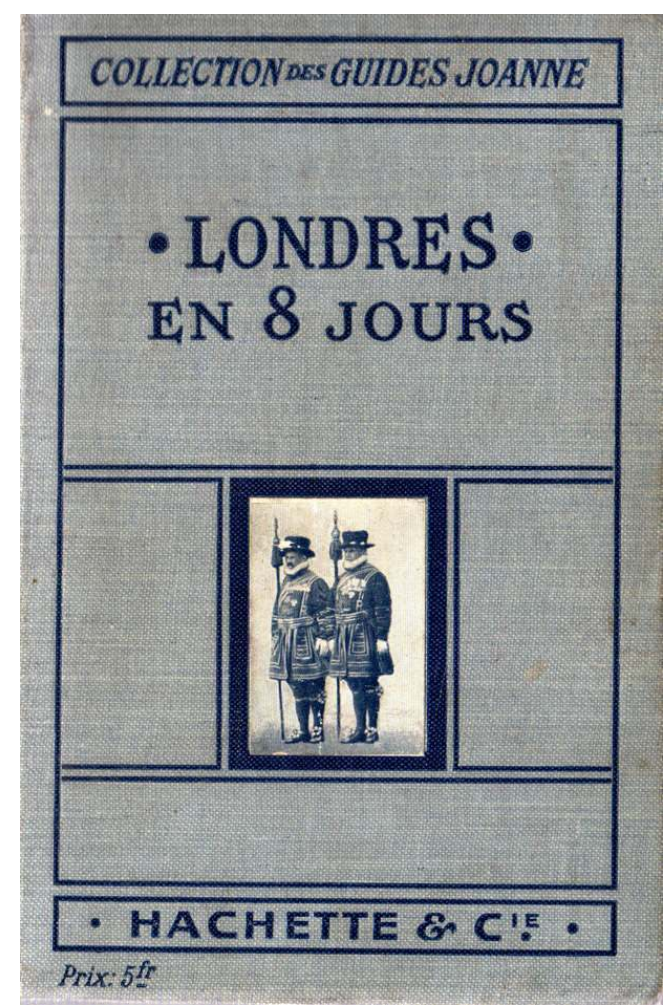

Guide Joanne illustré : Londres en 8 jours (1915). La couverture gris-bleu indique la langue de la publication : le français. 


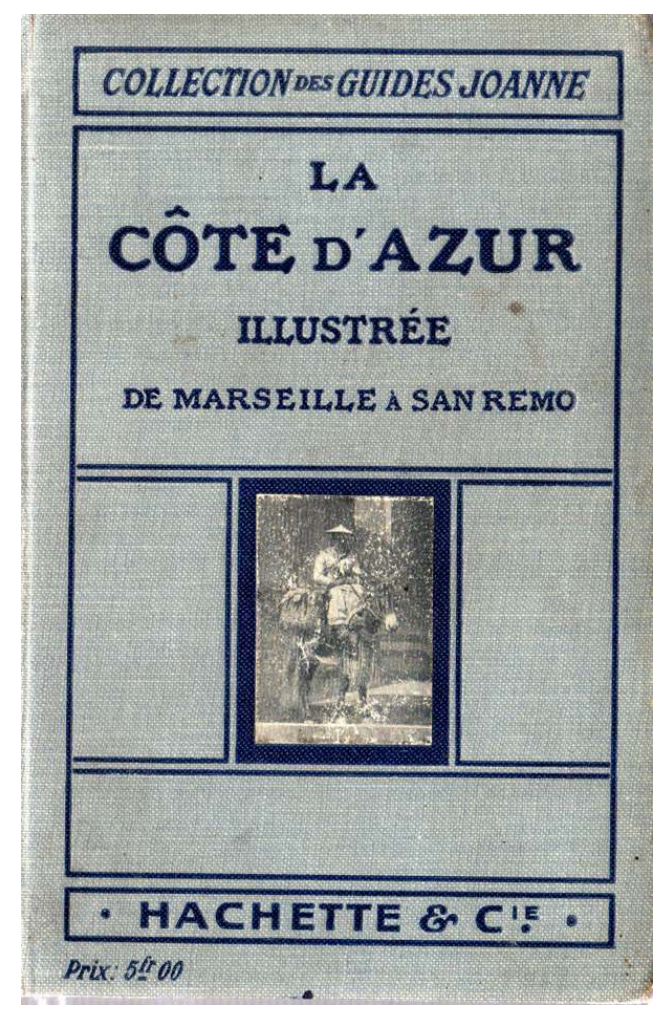

Guide Joanne illustré : La Côte d'Azur illustrée qui remplace la monographie cartonnée rouge (1913).

À partir de 1907, les «Guides-Joanne illustrés » voient le jour : il s'agit de volumes dont l'originalité repose sur un grand nombre de gravures puis de photographies qui concernent des destinations limitées dans l'espace: une ville (Paris, Londres, Le Caire) (fig. $\mathbf{n}^{\circ} \mathbf{2 1}$ ) ou une petite région touristique (le Pays basque, la Côte d'Azur, les châteaux de la Loire) que l'on pouvait visiter en automobile. Un "glissement» d'un titre d'une collection à une autre est repérable : le guide La Côte d'Azur existait dans la série des monographies rouges, il devient La Côte d'Azur illustrée avec une couverture bleu gris (fig. $\mathbf{n}^{\circ}$ 22), signe distinctif de cette série éditée en trois langues repérables par la couleur de la reliure : français (bleu gris), anglais (orange) (fig. $\mathbf{n}^{\circ} \mathbf{2 3}$ ), et allemand (vert) (fig. $\left.\mathbf{n}^{\circ} \mathbf{2 4}\right)$. De même, le guide Le Caire et ses environs n'est qu'un extrait du guide d'Égypte en trois volumes adapté à un lectorat moins exigeant : les plans spécialement dessinés par Max Herz Bey, conservateur du musée d'Art islamique du Caire, sont remplacés par des photographies, certes attrayantes, mais moins informatives sur le plan historique et architectural. Quant au séjour dans une capitale, il tend à se raccourcir comme l'annoncent les titres Londres [Paris ou Rome] en huit jours. Le temps de visite est rentabilisé grâce à des itinéraires de visite bien conçus qui ne laissent guère de place à la flânerie. On assiste à un changement de clientèle. Moins fortunés que les aristocrates et grands bourgeois, les touristes séjournaient moins longtemps mais désiraient voir le maximum de curiosités en un minimum de temps. C'est le rapport au temps qui est en cours de modification: les transports modernes ont permis d'atteindre rapidement des destinations lointaines, la baisse du prix des billets de train ou de bateau et le développement de l'hébergement ont rendu possible l'accès au tourisme à des classes moins privilégiées qui font des voyages ou des séjours plus courts. 
Figure 23

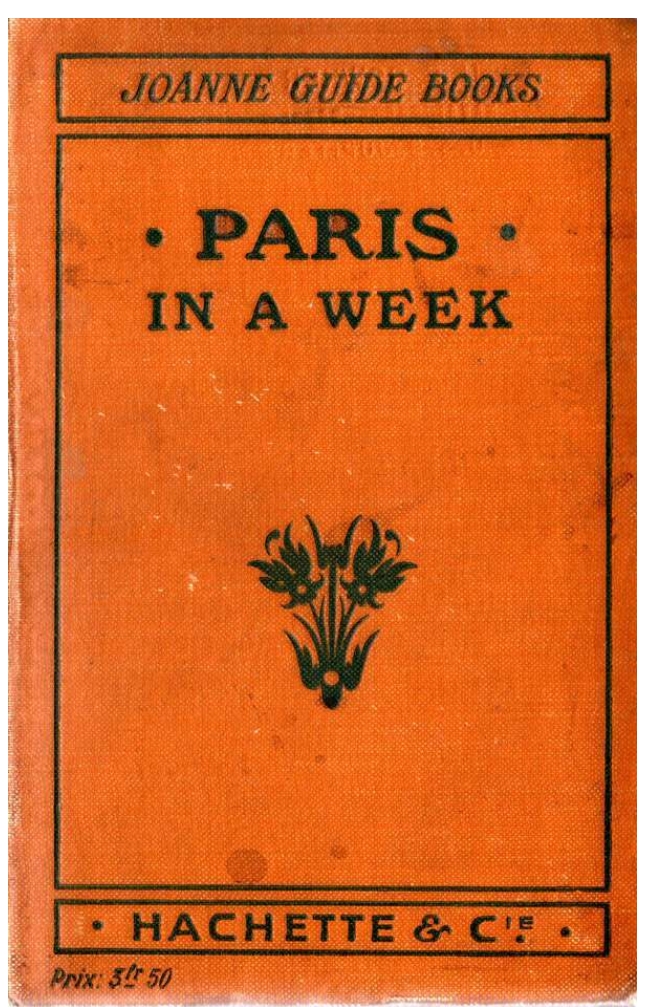

Guide Joanne illustré : Paris in a week (1910). La couverture orange indique la langue de la publication : I'anglais. 
Figure 24

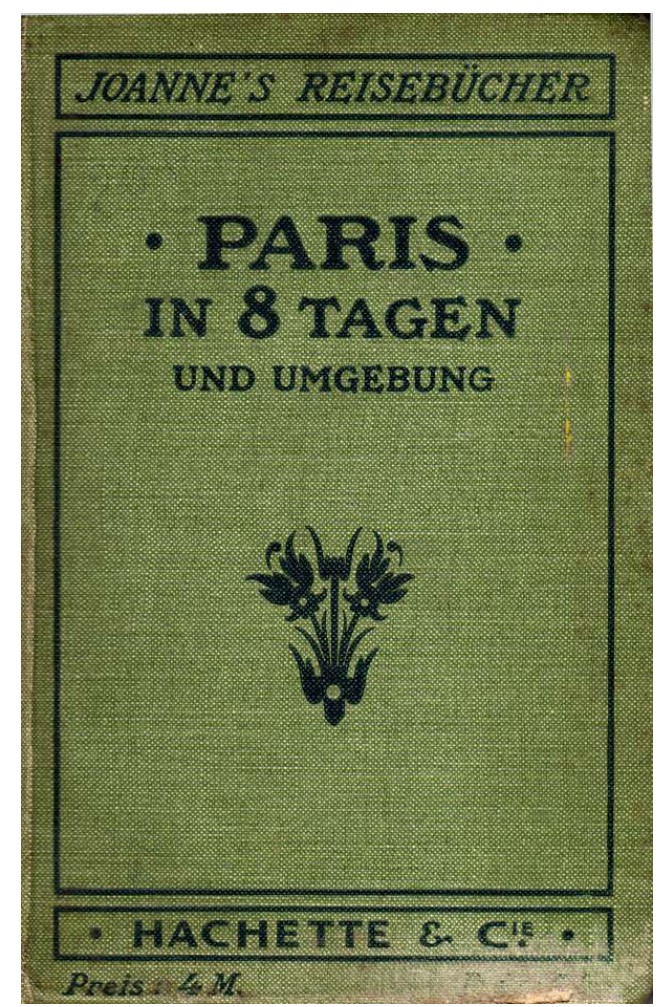

Guide Joanne illustré : Paris in 8 Tagen und Umgebung (1911). La couverture verte indique la langue de la publication : l'allemand.

42 La grande nouveauté est l'adaptation au tourisme automobile naissant : les Guides de l'IGF contiennent dès les premières années $d u \mathrm{XX}^{\mathrm{e}}$ siècle des cartes avec indication d'un circuit complété par un "profil », c'est-à-dire l'indication des dénivelés auxquels les véhicules (et surtout leurs freins) devront faire face (fig. $\mathbf{n}^{\circ} \mathbf{2 5}$ ).

D’autres guides simplifiés sont spécialement conçus pour ces nouveaux excursionnistes : dès 1910, des photographies fléchées permettaient aux visiteurs des châteaux de la Loire ${ }^{46}$ de choisir la bonne direction aux carrefours (fig. $\mathbf{n}^{\circ} \mathbf{2 6}$ ). Ils avaient à leur disposition des descriptions très précises des itinéraires, dignes des road books pour les concurrents de rallyes automobiles, la vitesse mise à part. Une nouvelle série intitulée explicitement « La route par l'image » était destinée aux automobilistes et aux cyclistes qui utilisaient ces moyens de transport pour leurs excursions autour de Paris dûment balisées par le guide ${ }^{47}$. 
Figure 25

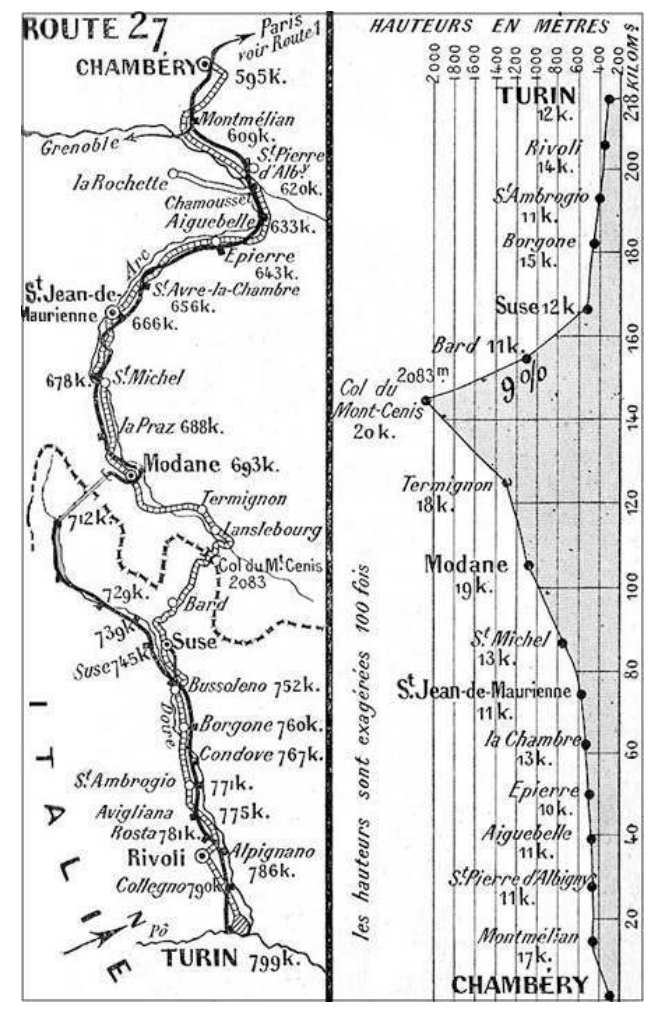

Un exemple d'itinéraire routier avec le profil correspondant (Savoie, 1908). 


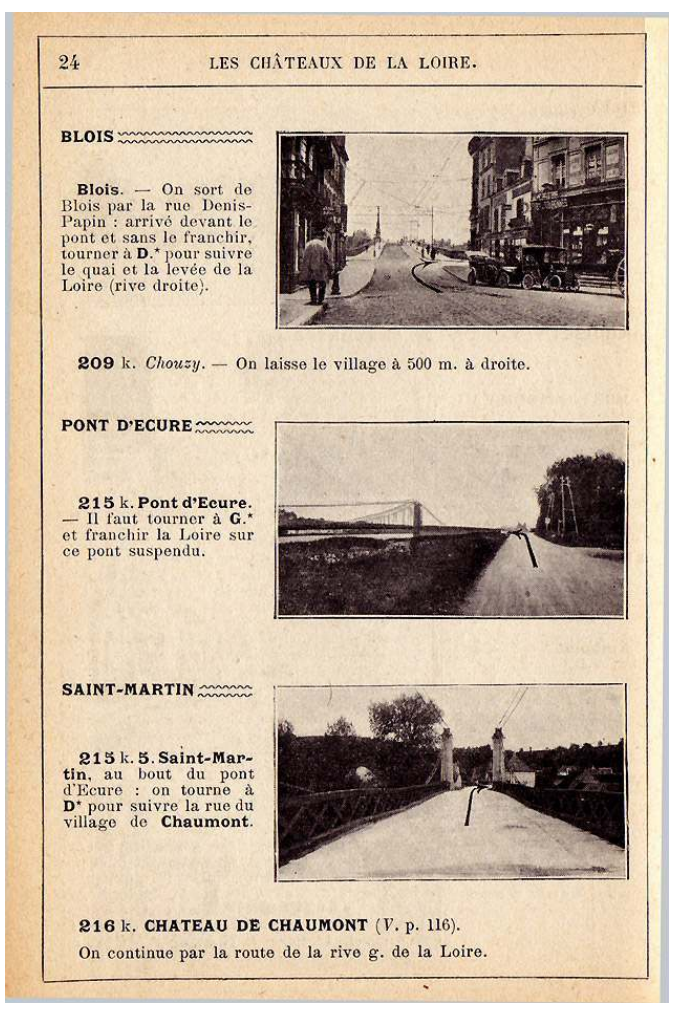

Un exemple d'itinéraire « par l'image » : les flèches indiquent la direction à prendre (Châteaux de la Loire, 1912).

L'atlas Sur Route ${ }^{48}$, paru vers 1906, permettait de consulter facilement des cartes routières en couleurs avec des renvois aux Guides Joanne pour les curiosités touristiques, l'Algérie en automobile $e^{49}$ (1911) suivait la même conception.

La diversification des collections est constante afin d'offrir au public des «nouveautés » qui n'en étaient pas toujours mais qui neutralisaient la concurrence intérieure et extérieure. Les diverses tentatives des éditeurs encore en lice après les rachats par Hachette et le monopole de la vente dans les gares n'ont jamais pu ébranler le géant: ainsi, Flammarion, Garnier et Conty ne furent que des outsiders. En revanche, l'arrivée en 1900 du guide gratuit de la firme Michelin qui disposait d'un réseau propre de distribution (revendeurs de carburant et mécaniciens) pouvait être envisagée comme dangereuse ${ }^{50}$. Il ne s'agissait au départ que d'un répertoire des garages pour les automobilistes, toutefois une riposte est anticipée avec les attractifs guides illustrés et leurs circuits détaillés. L'histoire montre que le danger était réel. À l'extérieur, la menace venait principalement de la concurrence des Guides Baedeker publiés en français, très populaires malgré leur prix équivalent et leur origine germanique. Cette menace ne prend fin qu'avec la victoire sur l'Allemagne en 1918. Il fallait donc proposer constamment des publications attractives qui correspondaient bien au public français et francophone ainsi qu'à l'évolution de ses goûts, pour voyager en France, terre de prédilection des Guides Joanne et dans une Europe aux routes bien balisées par des itinéraires qui avaient fait leurs preuves depuis plus d'un demi-siècle.

Lorsque Paul Joanne prend sa retraite en 1911, il laisse les Guides Joanne dans les mains de Marcel Monmarché qui avait été son collaborateur depuis 1890. Il s'agit donc de 
quelqu'un capable de transmettre les valeurs de la Librairie Hachette et de perpétuer l'esprit des collections de guides qui prend les rênes. En 1919, Marcel Monmarché assure le transfert des titres des Guides Joanne dans la nouvelle collection des Guides Bleus. Il applique la technique habituelle de l'habillage des contenus anciens par une couverture portant le nom de Guides Bleus. Grâce à cette transformation minime, il a assuré la transmission des règles mises au point par ses prédécesseurs et assuré le succès des Guides Joanne : la structure du plan et des contenus n'a guère varié et elle a été reprise par la plupart des guides de voyage modernes. C'est donc une collection née de la réunion de plusieurs fonds par Louis Hachette vers 1853 et baptisée Guides Joanne vers 1860 qui continue d'exister de nos jours sous le nom de Guides Bleus : bel exemple de longévité...

\section{NOTES}

1. - Hélène Morlier est ingénieur d'études, InVisu, USR 3103 CNRS/INHA. Auteur de la bibliographie des guides Joanne, elle prépare une thèse sur ce sujet sous la direction de M.-V. Ozouf-Marignier à l'ÉHESS.

2. - MISTLER, Jean. La Librairie Hachette de 1826 à nos jours. Paris : Hachette, 1964, p. 121-124. MOLLIER, Jean-Yves. Louis Hachette. Le fondateur d'un empire. 1870-1940. Paris : Fayard, 1999, p. 332 et suivantes.

3. - GUILCHER, Goulven. La rivalité Chaix-Hachette pour la conquête du marché de la lecture ferroviaire en France. Revue d'histoire des chemins de fer, 1992, HS 3, p. 279-305. PARINET, Élisabeth. Les bibliothèques de gare, un nouveau réseau pour le livre. Romantisme, 1993, 23/80, p. 95-106, part. p. 96.

4. - MISTLER, Jean. La Librairie Hachette de 1826 à nos jours. Paris : Hachette, 1964, p. 124. GUILCHER, Goulven, WITKOWSKI, Claude. La Bibliothèque des chemins de fer. Bulletin du Bibliophile, 1987, 4, p. 474-500, part. p. 478.

5. - CARMONA, Michel. Morny, le vice-empereur. Paris : Fayard, 2005, p. 97, 105, 245.

6. - MISTLER, Jean. La Librairie Hachette de 1826 à nos jours. Paris : Hachette, 1964, p. 125.

7. - IMEC, Fonds Hachette, HAC 83, Copie de traités I, 1844-1865, n9. Je remercie le professeur Jean-Yves Mollier de m'avoir signalé ces registres.

8. - Voyage de Paris à Corbeil et à Orléans (1853) et Enghien et la vallée de Montmorency (1853) : MORLIER, Hélène. Les Guides-Joanne, Genèse des Guides-Bleus. Paris : Les Sentiers débattus, $2007, n^{\circ} 143$ et 484 .

9. - Voyage de Paris à la mer et Guide à Londres furent réunis dans le Guide du voyageur à Londres (MORLIER, Hélène. Les Guides-Joanne, Genèse des Guides-Bleus. Paris : Les Sentiers débattus, 2007, n608).

10. - Sur les guides Richard, voir GUILCHER, Goulven. Les Guides-Richard I (1823-1836). La Lettre du marché Brassens, 26 janvier 1997, 54, p. 4-6. GUILCHER, Goulven. Les Guides-Richard II (1836-1855). La Lettre du marché Brassens, 26 mars 1997, 55, p. 4-7. MORLIER, Hélène. Les Guides-Joanne, Genèse des Guides-Bleus. Paris : Les Sentiers débattus, 2007, p. 32-33.

11. - IMEC, Fonds Hachette, HAC 83, Copie de traités I, 1844-1865, n77.

12. - JOANNE, Adolphe. Itinéraire descriptif et historique de la Suisse, du Jura français, de Baden-Baden et de la Forêt-Noire, de la chartreuse de Grenoble et des eaux d'Aix, du Mont Blanc, de la vallée de Chamouni, du Grand-St-Bernard et du Mont Rose, avec une carte 
routière imprimée sur toile, les armes de la Confédération suisse et des vingt-deux cantons, et deux grandes vues de la chaine du Mont-Blanc et des Alpes Bernoises. Paris : Paulin, 1841.

13. - EBEL, Johann Gottfried. Anleitung auf die nützlichste und genussvollste Art die Schweiz zu bereisen. Zürich : 1840 .

14. - MURRAY, John. Handbook for travellers in Switzerland and the Alps of Savoy and Piedmont. London : 1838.

15. - IMEC, Fonds Hachette, HAC $41.1, \mathrm{~F}^{\circ} 28 \mathrm{v} 2$, article 2.

16. - Jules Janin avait publié des récits de voyage ou des monographies (Voyage en Italie ou La Normandie chez Bourdin respectivement en 1842 et 1843) qui en faisaient un auteur tout désigné pour des guides.

17. - Journaliste et littérateur, F. Bernard est l'auteur d'une vingtaine de guides de la Bibliothèque des chemins de fer.

18. - Romancier et auteur dramatique, auteur d'une quinzaine de guides dans la même collection. 19. - Trois exemples des guides de la collection de la Bibliothèque des Chemins de Fer (fig. 5, 6, 7). On note les blasons des villes de départ et de terminus des lignes de chemin de fer : il s'agit ici des reliures cartonnées en percaline gaufrée qui étaient payées en supplément. Tous les guides existaient dans la version brochée rouge vermillon. Les titres seront peu à peu simplifiés pour plus de lisibilité.

20. - IMEC, Fonds Hachette, HAC 83, Copie de traités I, 1844-1865, n59, 15 juillet 1855.

21. - CARMONA, Michel. Morny, le vice-empereur. Paris : Fayard, 2005, p. 245.

22. - JANIN, Jules. De Paris à Dieppe, 1853 et 1855 (MORLIER, Hélène. Les Guides-Joanne, Genèse des Guides-Bleus. Paris : Les Sentiers débattus, 2007, nº161 et 162) et CHAPUS, Eugène. Dieppe et ses environs (Ibid., $\mathrm{n}^{\circ} 483$ ).

23. - IMEC, Fonds Hachette, HAC 83, Copie de traités I, 1844-1865, n¹91/1, décembre 1858.

24. - «Ce volume n'est point un livre de circonstance»: MONTÉMONT, Albert. Itinéraire descriptif de la Savoie, rapport de M. Albert Montémont sur cet ouvrage. Bulletin de la Société de géographie, $1860,4^{\mathrm{e}}$ série, $\mathrm{XX}$, p. 275-281.

25. - IMEC, Fonds Hachette, HAC 83, Copie de traités I, 1844-1865, n63/1, octobre 1855 et HAC $41.1, \mathrm{~F}^{\circ} 404$.

26. - IMEC, Fonds Hachette, HAC 83, Copie de traités I, 1844-1865, n¹87/1, décembre 1858.

27. - WEBER, Eugen. La France de nos aïeux. La fin des terroirs. Les imaginaires et la politique au XIX siècle. Paris : Fayard, 2005. VI. Des langues à foison (p. 93-123) et III. Le pied du roi (p. 47-59).

28. - Sic quoque docebo c'est-à-dire «Ainsi, j'enseignerai quand même ». MOLLIER, Jean-Yves. Louis Hachette. Paris : Fayard, 1999, p. 12.

29. - HINRICHSEN, A.W. Baedeker Katalog, Verzeichnis aller Baedeker-Reiseführer von 1832-1987. Holzminden : Ursula Hinrichsen Verlag, 1988, F 164 à F 193.

30. - LISTER, W.B.C. A bibliography of Murray's Handbooks for travellers and biographies of authors, editors, revisers and principal contributors. Dereham: Dereham Books, 1993, $\mathrm{n}^{\circ}$ 305-404.

31. - HINRICHSEN, A.W. Baedeker Katalog, Verzeichnis aller Baedeker-Reiseführer von 1832-1987. Holzminden : Ursula Hinrichsen Verlag, 1988, D 229a à D253.

32. - QUÉTIN. Guide du voyageur en Algérie, 1846 et BARBIER, J. Itinéraire historique et descriptif de l'Algérie, 1855 (MORLIER, Hélène. Les Guides-Joanne, Genèse des Guides-Bleus. Paris : Les Sentiers débattus, 2007, $\left.n^{\circ} 542-543\right)$.

33. - IMEC, Fonds Hachette, HAC 83, Copie de traités I, 1844-1865, n¹66, juillet 1858.

34. - MORLIER, Hélène. Les Guides-Joanne, Genèse des Guides-Bleus. Paris: Les Sentiers débattus, $2007, \mathrm{n}^{\circ} 1479$.

35. - Ibid., $\mathrm{n}^{\circ} 1457$. 


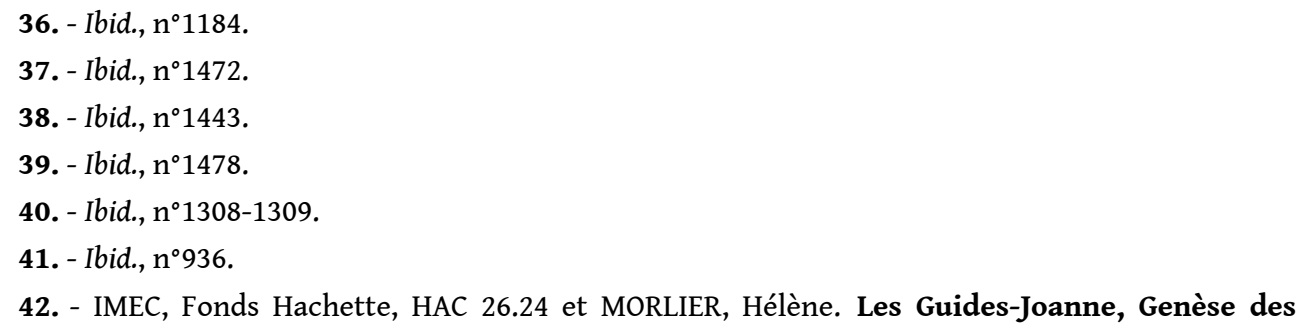

Guides-Bleus. Paris : Les Sentiers débattus, 2007, n 1477.

43. - Ibid., n¹458.

44. - Ibid., n749, paru en 1886.

45. - Ibid., n639-642, guides parus en 1888, 1893 et 1895.

46. - Ibid., $\mathrm{n}^{\circ} 1514$ puis $\mathrm{n}^{\circ} 1515-1517$.

47. - Ibid., $\mathrm{n}^{\circ} 1536-1537$.

48. - Ibid., $\mathrm{n}^{\circ} 1534$.

49. - Ibid., n¹534.

50. - Guide Michelin, offert gracieusement aux chauffeurs, édition 1900. Voir La saga du guide Michelin. Paris : Michelin, 2004 et FRANCON, Marc. Le guide Vert Michelin. L'invention du tourisme culturel populaire. Paris : Economica, 2001, p. 3-19.

\section{RÉSUMÉS}

Depuis la création des Guides Joanne, on peut souligner la remarquable adaptation aux nouveaux usages des touristes, aux changements et aux manières de voyager. Certaines destinations sont mises en valeur tandis que d'autres sont délaissées. Une analyse plus rigoureuse montre que les destinations et les différentes séries de guides sont étroitement liées à d'autres projets comme la construction de lignes de chemin de fer ou le développement des stations balnéaires et du tourisme automobile.

From the very beginning of the existence of the Joanne guide-books, one can notice the remarkable adaptation to new habits, changes and way of travelling. Even destinations could be favoured or become completely out of fashion. A deeper analysis of the destinations and different series shows that some guide-books were strongly linked to other projects like the building of new railway lines or the development of fashionable seaside resorts and motoring tourism.

\section{INDEX}

Mots-clés : Algérie, Baedeker, cartographie, chemin de fer, Empire ottoman, France, guides de voyage, Joanne, Murray, pays d'Europe, stations balnéaires, stations thermales, tourisme

\section{AUTEUR}

\section{HÉLĖNE MORLIER}

Ingénieur d'études, InVisu, USR 3103 CNRS/INHA,helene.morlier@inha.fr 